\title{
Molecular gradient for second-order Møller-Plesset perturbation theory using the divide-expand-consolidate (DEC) scheme
}

Kasper Kristensen, Poul Jørgensen, Branislav Jansík, Thomas Kjærgaard, and Simen Reine

Citation: The Journal of Chemical Physics 137, 114102 (2012); doi: 10.1063/1.4752432

View online: http://dx.doi.org/10.1063/1.4752432

View Table of Contents: http://aip.scitation.org/toc/jcp/137/11

Published by the American Institute of Physics

\section{Articles you may be interested in}

Linear scaling coupled cluster method with correlation energy based error control

The Journal of Chemical Physics 133, 014107 (2010); 10.1063/1.3456535

Analytical energy gradients for local second-order Møller-Plesset perturbation theory using density fitting approximations

The Journal of Chemical Physics 121, 737 (2004); 10.1063/1.1760747

The divide-expand-consolidate family of coupled cluster methods: Numerical illustrations using second order Møller-Plesset perturbation theory

The Journal of Chemical Physics 136, 014105 (2012); 10.1063/1.3667266

The molecular gradient using the divide-expand-consolidate resolution of the identity second-order MøllerPlesset perturbation theory: The DEC-RI-MP2 gradient

The Journal of Chemical Physics 145, 024106 (2016); 10.1063/1.4956454

Long-range interactions from the many-pair expansion: A different avenue to dispersion in DFT

The Journal of Chemical Physics 146, 024111 (2017); 10.1063/1.4973728

Incremental full configuration interaction

The Journal of Chemical Physics 146, 104102 (2017); 10.1063/1.4977727

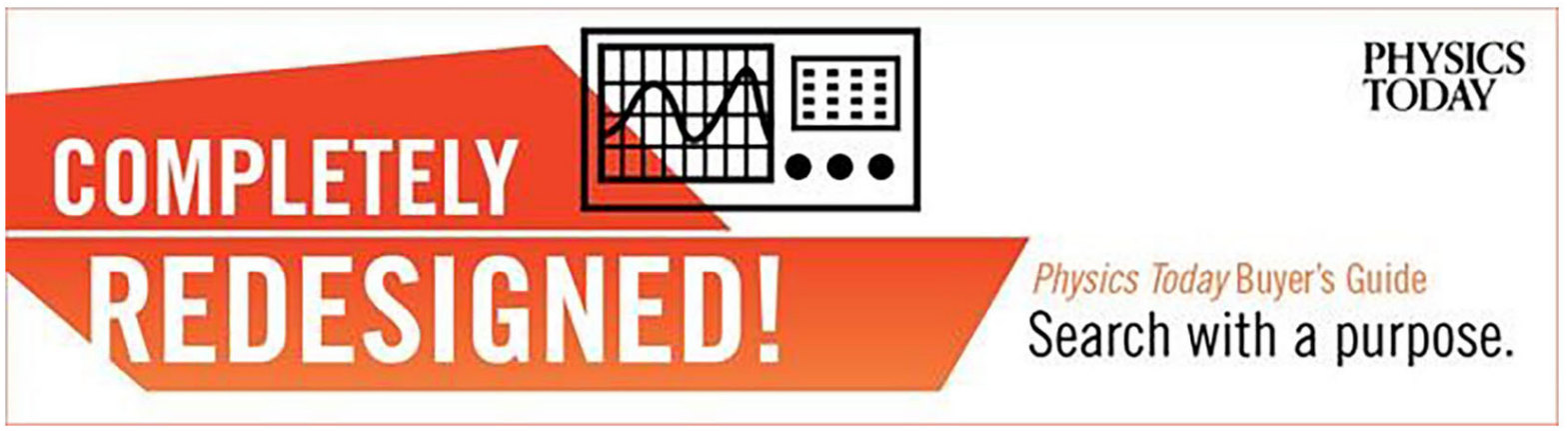




\title{
Molecular gradient for second-order Møller-Plesset perturbation theory using the divide-expand-consolidate (DEC) scheme
}

\author{
Kasper Kristensen, ${ }^{1}$ Poul Jørgensen, ${ }^{1}$ Branislav Jansík, ${ }^{1}$ Thomas Kjærgaard, ${ }^{2}$ \\ and Simen Reine ${ }^{2}$ \\ ${ }^{1}$ Lundbeck Foundation Center for Theoretical Chemistry, Department of Chemistry, Aarhus University, \\ Langelandsgade 140, DK-8000 Aarhus C, Denmark \\ ${ }^{2}$ Centre for Theoretical and Computational Chemistry, Department of Chemistry, Oslo university, \\ Postbox 1033, Blindern, 0315 Oslo, Norway
}

(Received 29 April 2012; accepted 30 August 2012; published online 17 September 2012)

\begin{abstract}
We demonstrate that the divide-expand-consolidate (DEC) scheme - which has previously been used to determine the second-order Møller-Plesset (MP2) correlation energy - can be applied to evaluate the MP2 molecular gradient in a linear-scaling and embarrassingly parallel manner using a set of local Hartree-Fock orbitals. All manipulations of four-index quantities (describing electron correlation effects) are carried out using small local orbital fragment spaces, whereas two-index quantities are treated for the full molecular system. The sizes of the orbital fragment spaces are determined in a black-box manner to ensure that the error in the DEC-MP2 correlation energy compared to a standard MP2 calculation is proportional to a single input threshold denoted the fragment optimization threshold (FOT). The FOT also implicitly controls the error in the DEC-MP2 molecular gradient as substantiated by a theoretical analysis and numerical results. The development of the DEC-MP2 molecular gradient is the initial step towards calculating higher order energy derivatives for large molecular systems using the DEC framework, both at the MP2 level of theory and for more accurate coupledcluster methods. ( 2012 American Institute of Physics. [http://dx.doi.org/10.1063/1.4752432]
\end{abstract}

\section{INTRODUCTION}

During the last decades much attention has been devoted towards developing coupled-cluster (CC) methods with low-order computational scaling to extend the applicability range of $\mathrm{CC}$ to include large molecular systems. Following the pioneering work on local CC methods by Pulay, ${ }^{1}$ and Saeb $\varnothing$ and Pulay, ${ }^{2}$ the local coupled-cluster method of Hampel and Werner ${ }^{3}$ and Schütz and Werner ${ }^{4-6}$ was developed. Many other methods for obtaining reduced scaling have been proposed, ${ }^{7-26}$ including atomicorbital-based $\mathrm{CC},{ }^{7-9}$ the natural-linear-scaling approach, ${ }^{11}$ the cluster-in-molecule approach, ${ }^{12-14}$ the divide-and-conquer approach, ${ }^{15}$ the fragment-molecular-orbital approach, ${ }^{16}$ the incremental scheme, ${ }^{17,18}$ Laplace-MP2, ${ }^{19-21}$ the dynamically screened local correlation method, ${ }^{22}$ local pair natural orbital approximations, ${ }^{23,24}$ and orbital-specific virtual orbital approximations. ${ }^{25,26}$

We have recently proposed the linear-scaling and embarrassingly parallel divide-expand-consolidate (DEC) CC approach for evaluating the CC energy. ${ }^{27-29}$ An important prerequisite for DEC is the existence of a set of local HartreeFock (HF) orbitals. We use our orbital localization strategy, ${ }^{30}$ where powers of the orbital variances are minimized to generate a set of local orthonormal HF orbitals both for the occupied and unoccupied orbital spaces. In the DEC scheme the standard CC calculation is divided into a set of independent $\mathrm{CC}$ fragment calculations each using their own subset of the total orbital space. This is possible because both occupied and unoccupied HF orbitals are local. A crucial feature of the
DEC method is that the orbital fragment spaces are systematically expanded in a black-box manner during the calculation to ensure that the fragment energies are determined to a preset threshold denoted the fragment optimization threshold (FOT). This, in turn, defines the total CC correlation energy as a sum of the fragment energies to a preset threshold compared to a standard molecular calculation.

The determination of molecular equilibrium structures i.e., local minima on the potential energy surface - is one of the most important tasks of electronic structure theory. An efficient geometry optimization requires that molecular gradients are evaluated analytically. The analytic evaluation of molecular gradients was initiated by the seminal work of Pulay $^{31}$ on the HF molecular gradient. Following this work, gradients for correlated wave function methods have been developed - including MP $2,{ }^{32} \mathrm{MP} 3,{ }^{33} \mathrm{MP} 4,{ }^{34}$ configuration interaction (CI), ${ }^{35-38}$ and $\mathrm{CC}^{39-42}$ gradients. In this work our focus is on the MP2 molecular gradient within the DEC framework.

Formulations of the MP2 molecular gradient in the molecular orbital (MO) basis ${ }^{32,43-54}$ are prone to high-order computational scaling with system size and can therefore not be used for calculations on large molecular systems, although efficient parallelization extend the applicability range $.^{55} \mathrm{How}-$ ever, MP2 gradients for very large molecules are out of reach using the traditional $\mathrm{MO}$ formulation.

Different approaches have been attempted for reducing the high-order scaling of traditional MP2 gradient formulations. In the MP2 resolution of the identity (MP2-RI) 
approach, ${ }^{56,57}$ where two-electron integrals are expanded in an auxiliary basis, reduced scaling has been obtained for the MP2 gradient. ${ }^{58-61}$ A different strategy proposed by Schweizer, Doser, and Ochsenfeld ${ }^{62}$ is to abandon the MO basis and reformulate the MP2 gradient equations in the atomic orbital (AO) basis using Laplace transformations of the energy denominator. ${ }^{63}$ In a local CC context Werner and coworkers have presented molecular gradients for the local MP2 energy ${ }^{64}$ and also extended this approach to employ densityfitting techniques. ${ }^{65}$ The MP2 molecular gradient has also recently been presented for the fragment-molecular-orbital method. ${ }^{66}$

In this paper we describe how the MP2 molecular gradient may be evaluated within the DEC framework by generalizing the DEC Lagrangian correlation energy scheme presented in Ref. 29. Using a set of local orthogonal occupied and virtual orbitals ${ }^{30}$ we demonstrate that all intermediates involving four-index quantities (amplitudes, amplitude multipliers, and two-electron integrals) in the full molecular MP2 gradient expression can be evaluated and contracted in terms of small local orbital fragment calculations. Only two-index quantities, such as effective MP2 densities, need to be stored for the full molecular system. Following the DEC fragment calculations the orbital rotation multipliers (two indices) are determined by solving a set of coupled-perturbed (CP) HF equations for the full molecular system, and the full MP2 gradient is subsequently calculated by contracting full molecular two-index quantities in the AO basis with differentiated integrals. The FOT - which controls the error of the DECMP2 Lagrangian correlation energy by optimizing the sizes of the orbital fragment spaces in a black-box manner - also implicitly controls the precision of the DEC-MP2 gradient. Although limiting ourselves to the MP2 model in this work, we note that the presented formulation serves as a precursor for obtaining molecular gradients for higher order CC models using the DEC scheme.

The correlated part (atomic fragment and atomic pair fragment calculations) of the DEC-MP2 molecular gradient scheme is linear-scaling and embarrassingly parallelizable, since distant atomic pairs describing vanishing dispersion interactions may be neglected, and since the fragment calculations are independent. Thus, if linear-scaling techniques ${ }^{67-73}$ are also applied for the HF-like part of the calculation (the preceding HF calculation, the solution of the $\mathrm{CP} \mathrm{HF}$ equation, and the final contractions of effective densities with differentiated integrals), the calculation of the MP2 molecular gradient as a whole is linear-scaling.

Let us comment on the scope of this work and the DEC method in general. The main purpose of the DEC scheme is not to compete with a standard implementation for small and medium-sized molecules. Rather, the DEC scheme is an attempt to enable MP2 calculations (more generally coupledcluster calculations) on large molecular systems where standard implementations encounter a scaling wall (both memoryand time-wise). Although the treatment of large molecules is the focus area for the DEC method, it is also crucial to verify that the DEC scheme is able to reproduce the results of a standard calculation to any requested tolerance (the FOT) in a black box manner. The core of the present paper is the theoretical development which demonstrates how and why the DEC scheme can be used to reproduce the standard MP2 gradient to a given precision in a linear-scaling and embarrassingly parallel manner. To support the theoretical analysis we provide some numerical results on medium-sized systems (30-50 atoms) which verify that the DEC-MP2 molecular gradient reproduces the standard MP2 gradient to the expected precision. Such investigations are of course necessary before the DEC scheme can routinely be applied for larger molecular systems.

The paper is structured as follows. In Sec. II we derive the full molecular MP2 gradient equations in a general form which is convenient for developing the DEC-MP2 gradient scheme in Sec. III. Section IV contains numerical results, and in Sec. V we give some concluding remarks and future perspectives.

\section{FULL CALCULATION: THE MP2 MOLECULAR GRADIENT}

The equations for the MP2 molecular gradient in the canonical MO basis have been discussed by several authors. ${ }^{32,43-54}$ However, to be able to apply the DEC scheme to evaluate the MP2 gradient, it is necessary to express the MP2 gradient in terms of a set of local HF orbitals and thus abandon any reference to the canonical basis. In this section we derive a general expression for the MP2 gradient assuming only that the HF orbitals at the reference geometry are orthonormal and that they represent an optimized HF wave function, i.e., that the occupied-virtual (and virtual-occupied) block of the Fock matrix is zero. The resulting gradient expression is written in a specific form which, for local HF orbitals ${ }^{30}$ is straightforwardly extendable to the DEC scheme as will be outlined in Sec. III.

\section{A. MP2 energy}

We limit the scope of this paper to closed-shell molecules and assume real orbitals. We use the index convention that $i$, $j, k, l$ denote occupied MOs, $a, b, c, d$ denote virtual MOs, $p$, $q, r, s, t, u$ denote general MOs (occupied or virtual), while Greek letters $\mu, v, \alpha, \beta, \gamma, \delta$ denote AOs.

The MP2 energy at a general geometry $\mathbf{x}$ may be written as a sum of a HF contribution $E_{\mathrm{HF}}(\mathbf{x})$ and a correlation contribution $E_{\text {corr }}(\mathbf{x}),{ }^{74}$

$$
\begin{gathered}
E_{\mathrm{MP} 2}(\mathbf{x})=E_{\mathrm{HF}}(\mathbf{x})+E_{\mathrm{corr}}(\mathbf{x}), \\
E_{\mathrm{HF}}(\mathbf{x})=2 \sum_{i} h_{i i}(\mathbf{x})+\sum_{i j} L_{i i j j}(\mathbf{x})+h_{\mathrm{nuc}}(\mathbf{x}), \\
E_{\mathrm{corr}}(\mathbf{x})=\sum_{a i b j} t_{i j}^{a b}(\mathbf{x}) L_{a i b j}(\mathbf{x}) .
\end{gathered}
$$

The one-electron integral $h_{p q}(\mathbf{x})$ is given by

$$
\begin{aligned}
& h_{p q}(\mathbf{x})=\left\langle\phi_{p}(\mathbf{x})\left|h_{1}(\mathbf{x})\right| \phi_{q}(\mathbf{x})\right\rangle, \\
& h_{1}(\mathbf{x})=-\frac{1}{2} \nabla^{2}-\sum_{K} Z_{K} r_{K}^{-1},
\end{aligned}
$$


where $Z_{K}$ is the charge of nucleus $K$ and $r_{K}$ is the distance from the electronic integration coordinate to nucleus $K$ (which ultimately depends on the specific molecule geometry $\mathbf{x}$ ), and $L_{\text {pqrs }}(\mathbf{x})$ is expressed in terms of two-electron integrals $g_{\text {pqrs }}(\mathbf{x})$ (using Mulliken notation) according to

$$
\begin{gathered}
L_{p q r s}(\mathbf{x})=2 g_{p q r s}(\mathbf{x})-g_{p s r q}(\mathbf{x}), \\
g_{p q r s}(\mathbf{x})=\left(\phi_{p}(\mathbf{x}) \phi_{q}(\mathbf{x}) \mid \phi_{r}(\mathbf{x}) \phi_{s}(\mathbf{x})\right) .
\end{gathered}
$$

Finally, $h_{\text {nuc }}(\mathbf{x})$ in Eq. (2) is the nuclear-nuclear repulsion energy. The MOs $\{\phi\}$ entering Eq. (1) are orthonormal and satisfy the HF optimization condition, i.e.,

$$
\begin{aligned}
& \left\langle\phi_{p}(\mathbf{x}) \mid \phi_{q}(\mathbf{x})\right\rangle=\delta_{p q}, \\
& F_{a i}(\mathbf{x})=F_{i a}(\mathbf{x})=0,
\end{aligned}
$$

where the Fock matrix is given by

$$
F_{p q}=h_{p q}+\sum_{i} L_{p q i i}
$$

The MP2 amplitudes $t$ are determined from the MP2 amplitude equations, ${ }^{74}$

$$
\begin{aligned}
\Omega_{i j}^{a b}(\mathbf{x})= & g_{a i b j}(\mathbf{x}) \\
& +\sum_{c}\left(t_{i j}^{c b}(\mathbf{x}) F_{a c}(\mathbf{x})+t_{i j}^{a c}(\mathbf{x}) F_{b c}(\mathbf{x})\right) \\
& -\sum_{k}\left(t_{k j}^{a b}(\mathbf{x}) F_{k i}(\mathbf{x})+t_{i k}^{a b}(\mathbf{x}) F_{k j}(\mathbf{x})\right)=0
\end{aligned}
$$

where the amplitudes satisfy the symmetry relation $t_{i j}^{a b}=t_{j i}^{b a}$.

\section{B. Orbital bases}

Consider a molecular system at some reference geometry $\mathbf{x}_{0}$. The optimized MOs $\phi\left(\mathbf{x}_{0}\right)$ are expanded in terms of atomic orbitals $\chi\left(\mathbf{x}_{0}\right)$ :

$$
\phi_{p}\left(\mathbf{x}_{0}\right)=\sum_{\mu} \chi_{\mu}\left(\mathbf{x}_{0}\right) C_{\mu p}\left(\mathbf{x}_{0}\right) .
$$

The MOs are orthonormal

$$
\left\langle\phi_{p}\left(\mathbf{x}_{0}\right) \mid \phi_{q}\left(\mathbf{x}_{0}\right)\right\rangle=\delta_{p q}
$$

and, since the MOs are optimized, the occupied-virtual and virtual-occupied blocks of the Fock matrix are zero,

$$
F_{a i}\left(\mathbf{x}_{0}\right)=F_{i a}\left(\mathbf{x}_{0}\right)=0 .
$$

When the geometry is changed from $\mathbf{x}_{0} \rightarrow \mathbf{x}$, the MO coefficients change to ensure that Eqs. (8) and (9) are satisfied.

To describe the change in the MOs as the geometry changes we first introduce a set of so-called unmodified molecular orbitals (UMOs) $\tilde{\phi}(\mathbf{x})$ expressed in terms of the optimized MO coefficients at the reference geometry $\mathbf{x}_{0},{ }^{75}$

$$
\tilde{\phi}_{p}(\mathbf{x})=\sum_{\mu} \chi_{\mu}(\mathbf{x}) C_{\mu p}\left(\mathbf{x}_{0}\right) .
$$

The UMOs are not orthogonal (except at $\mathbf{x}=\mathbf{x}_{0}$ ). It is therefore convenient to introduce a connection matrix ${ }^{76} \mathbf{T}(\mathbf{x})$ which makes the MOs orthonormal at all geometries. We thus introduce an orthonormalized molecular orbital (OMO) basis $\hat{\phi}(\mathbf{x})$,

$$
\begin{gathered}
\hat{\phi}_{p}(\mathbf{x})=\sum_{t} \tilde{\phi}_{t}(\mathbf{x}) T_{t p}(\mathbf{x}), \\
\left\langle\hat{\phi}_{p}(\mathbf{x}) \mid \hat{\phi}_{q}(\mathbf{x})\right\rangle=\left(\mathbf{T}^{\mathrm{T}}(\mathbf{x}) \tilde{\mathbf{S}}(\mathbf{x}) \mathbf{T}(\mathbf{x})\right)_{p q}=\delta_{p q},
\end{gathered}
$$

where $\tilde{\mathbf{S}}(\mathbf{x})$ is the overlap matrix in the UMO basis. Finally, the optimized MOs $\phi(\mathbf{x})$ at a general geometry $\mathbf{x}$ can be written as a unitary transformation $\mathbf{U}(\mathbf{x})$ of the OMOs

$$
\phi_{p}(\mathbf{x})=\sum_{t} \hat{\phi}_{t}(\mathbf{x}) U_{t p}(\mathbf{x}), \quad \mathbf{U}^{\mathrm{T}}(\mathbf{x}) \mathbf{U}(\mathbf{x})=\mathbf{1} .
$$

Assuming real orbitals, the unitary transformation matrix $\mathbf{U}(\mathbf{x})$ may be parameterized in terms of an anti-symmetric real matrix $\kappa(\mathbf{x})$,

$$
\mathbf{U}(\mathbf{x})=\mathrm{e}^{-\kappa(\mathbf{x})}, \quad \kappa(\mathbf{x})=-\kappa^{\mathrm{T}}(\mathbf{x}) .
$$

Introducing the OMO basis leads to separate orbital reorthonormalization effects (described by the $\mathbf{T}(\mathbf{x})$ matrix) and orbital relaxation contributions (described by the $\boldsymbol{\kappa}(\mathbf{x})$ matrix) for energy derivatives. ${ }^{75,77}$ An infinite number of connection matrices T(x) exist which satisfy Eq. (17). The different connection matrices lead to different reorthonormalization contributions and thus to different relaxation contributions as the energy derivatives do not depend on the choice of connection matrix. In a local correlation context the connection matrix should be chosen to conserve locality when the energy derivatives are evaluated. The OMOs at a displaced geometry $\mathbf{x}_{0}+\delta \mathbf{x}$ should thus be chosen to resemble the local HF orbitals at the reference geometry $\mathbf{x}_{0}$ as closely as possible, i.e., the smallest possible connection matrix from local orbitals $\phi\left(\mathbf{x}_{0}\right)$ to OMOs $\hat{\phi}\left(\mathbf{x}_{0}+\delta \mathbf{x}\right)$ should be chosen. The smallest connection matrix in a least squares sense is obtained using the natural connection, ${ }^{76}$ which, in general, is the optimal choice when energy derivatives are evaluated using local correlation methods. However, for the molecular gradient only orbital reorthonormalization effects are needed (contributions involving the first order $\boldsymbol{\kappa}^{(1)}(\mathbf{x})$ matrix do not enter the molecular gradient expression, see Sec. II D), and the same final expression is obtained, independently of the choice of connection matrix. For simplicity in the derivation of the molecular MP2 gradient we have therefore used the symmetric Löwdin OMO connection,

$$
\mathbf{T}(\mathbf{x})=\tilde{\mathbf{S}}^{-\frac{1}{2}}(\mathbf{x}),
$$

which corresponds to choosing the set of OMOs which is closest to the UMOs at the displaced geometry. We emphasize, however, that for second- and higher-order molecular properties the natural connection matrix ${ }^{76}$ is the optimal choice in a local correlation context.

Let us summarize the different orbital bases that have been introduced. In general, quantities in the MO basis are denoted with small Roman letters and using the same identifier as the basis in which they are expressed, while quantities in the AO basis are denoted with Greek letters. For example, an element of the overlap matrix in the AO, UMO, OMO, and 
optimized bases can be written as

$$
\begin{gathered}
S_{\mu \nu}(\mathbf{x})=\left\langle\chi_{\mu}(\mathbf{x}) \mid \chi_{\nu}(\mathbf{x})\right\rangle \quad \text { (AO basis), } \\
\tilde{S}_{p q}(\mathbf{x})=\left\langle\tilde{\phi}_{p}(\mathbf{x}) \mid \tilde{\phi}_{q}(\mathbf{x})\right\rangle \quad \text { (UMO basis), } \\
\hat{S}_{p q}(\mathbf{x})=\left\langle\hat{\phi}_{p}(\mathbf{x}) \mid \hat{\phi}_{q}(\mathbf{x})\right\rangle \quad \text { (OMO basis), } \\
S_{p q}(\mathbf{x})=\left\langle\phi_{p}(\mathbf{x}) \mid \phi_{q}(\mathbf{x})\right\rangle \quad \text { (optimized basis). }
\end{gathered}
$$

We are now ready to set up the MP2 Lagrangian which directly provides us with an expression for the MP2 molecular gradient.

\section{MP2 Lagrangian}

The molecular gradient could now be determined by straightforward differentiation of the MP2 energy in Eq. (1). However, since the MP2 energy is not variational, the resulting expression would involve first-order MO coefficients and first order MP2 amplitudes. To avoid the determination of first-order parameters it is therefore convenient to introduce a variational MP2 Lagrangian ${ }^{46}$ where the MP2 amplitude equation in Eq. (11) and the HF optimization condition in Eq. (9) are added as constraints with associated multipliers $\bar{t}$ and $\bar{\kappa}$, respectively,

$$
\begin{aligned}
L_{\mathrm{MP} 2}(\mathbf{x}, \kappa, t, \bar{t}, \bar{\kappa})= & E_{\mathrm{MP} 2}(\mathbf{x})+\frac{1}{2} \sum_{a i b j} \bar{t}_{i j}^{a b}(\mathbf{x}) \Omega_{i j}^{a b}(\mathbf{x}) \\
& +2 \sum_{a i} \bar{\kappa}_{a i}(\mathbf{x}) F_{a i}(\mathbf{x})
\end{aligned}
$$

Note that it is not necessary to add the orthonormalization condition for the MOs as a constraint in the Lagrangian, since this condition is implicitly taken care of by expressing the optimized orbitals in terms of the OMOs, see Eqs. (16)-(18). Also note that it is not necessary to add multipliers $\bar{\kappa}_{i j}$ and $\bar{\kappa}_{a b}$ associated with locality equations for the occupied and virtual orbitals, respectively, since the Lagrangian is invariant with respect to rotations among the occupied orbitals and among the virtual orbitals. In other words, the use of the rather intricate connection scheme introduced in Sec. II B avoids the need to include an additional localization criterion to the Lagrangian. This is convenient since no localization multiplier equation need to be solved, and no differentiated localization equation need to be calculated for the gradient. The connection scheme thus ensures that any set of local orbitals may be used without adapting the MP2 gradient to the specific localization function used. As discussed above, for second- and higher-order properties the use of the natural connection ${ }^{76}$ will ensure that the locality of the local orbitals for the unperturbed reference system is retained as closely as possible when the system is perturbed.

The multipliers are determined such that the Lagrangian is variational with respect to all parameters - i.e., the amplitudes, the MO coefficients, and the associated multipliers:

$$
\frac{\partial L_{\mathrm{MP} 2}(\mathbf{x}, \kappa, t, \bar{t}, \bar{\kappa})}{\partial t_{i j}^{a b}(\mathbf{x})}=0,
$$

$$
\begin{aligned}
& \frac{\partial L_{\mathrm{MP} 2}(\mathbf{x}, \kappa, t, \bar{t}, \bar{\kappa})}{\partial \bar{t}_{i j}^{a b}(\mathbf{x})}=0, \\
& \frac{\partial L_{\mathrm{MP} 2}(\mathbf{x}, \kappa, t, \bar{t}, \bar{\kappa})}{\partial \kappa_{d l}(\mathbf{x})}=0, \\
& \frac{\partial L_{\mathrm{MP} 2}(\mathbf{x}, \kappa, t, \bar{t}, \bar{\kappa})}{\partial \bar{\kappa}_{d l}(\mathbf{x})}=0 .
\end{aligned}
$$

Equations (27) and (29) trivially reproduce Eqs. (11) and (9), respectively, while Eqs. (26) and (28) yield equations for the $\bar{t}$ and $\bar{\kappa}$ multipliers, respectively. The specific forms of these equations are given in Sec II E.

Using the variational criteria in Eqs. (26)-(29) the MP2 gradient at the reference geometry $\mathbf{x}=\mathbf{x}_{0}$ (total derivative of $\left.E_{\mathrm{MP} 2}(\mathbf{x})\right)$ may now be determined as a partial derivative of the Lagrangian in Eq. (25) (omitting function arguments for the sake of brevity),

$$
\left.\frac{d E_{\mathrm{MP} 2}}{d \mathbf{x}}\right|_{\mathbf{x}=\mathbf{x}_{0}}=\left.\frac{\partial L_{\mathrm{MP} 2}}{\partial \mathbf{x}}\right|_{\mathbf{x}=\mathbf{x}_{0}} \equiv L_{\mathrm{MP} 2}^{(x)} .
$$

In the following, the notation $A^{(x)}$ will be adapted for the partial derivative of any quantity $A$ with respect to $\mathbf{x}$ evaluated at $\mathbf{x}=\mathbf{x}_{0}$. Using this notation and Eq. (25), we may write Eq. (30) as

$$
\begin{aligned}
L_{\mathrm{MP} 2}^{(x)}= & E_{\mathrm{MP} 2}^{(x)}+\frac{1}{2} \sum_{a i b j} \bar{t}_{i j}^{a b}\left(\mathbf{x}_{0}\right) \Omega_{i j}^{a b,(x)} \\
& +2 \sum_{a i} \bar{\kappa}_{a i}\left(\mathbf{x}_{0}\right) F_{a i}^{(x)}
\end{aligned}
$$

where only the terms in $\Omega_{i j}^{a b,(x)}$ and $F_{a i}^{(x)}$, which depends explicitly on $\mathbf{x}$ (not implicitly via any of the parameters) contribute. In summary, no first order wave function parameters occur in Eq. (31) at the expense that zeroth order multipliers $\bar{t}\left(\mathbf{x}_{0}\right)$ and $\bar{\kappa}\left(\mathbf{x}_{0}\right)$ need to be determined.

\section{Expression for the molecular gradient}

Considering Eq. (31) we note that only partial geometry derivatives at $\mathbf{x}=\mathbf{x}_{0}$ are required. In particular, no terms involving geometry derivatives of the $\mathbf{U}$ matrix in Eq. (18) contribute to Eq. (31). Thus, the partial geometry derivative of an optimized orbital $\phi_{p}(\mathbf{x})$ in Eq. (18) (evaluated at the reference geometry) effectively equals the partial geometry derivative of the OMO $\hat{\phi}_{p}(\mathbf{x})$,

$$
\begin{aligned}
\phi_{p}^{(x)} & =\left.\frac{\partial \phi_{p}(\mathbf{x})}{\partial \mathbf{x}}\right|_{\mathbf{x}=\mathbf{x}_{0}}=\left.\sum_{t} \frac{\partial \hat{\phi}_{t}(\mathbf{x})}{\partial \mathbf{x}}\right|_{\mathbf{x}=\mathbf{x}_{0}} U_{t p}\left(\mathbf{x}_{0}\right) \\
& =\sum_{t} \hat{\phi}_{t}^{(x)} \delta_{t p}=\hat{\phi}_{p}^{(x)}
\end{aligned}
$$

where we have used that, by construction, $\mathbf{U}(\mathbf{x})$ becomes the identity matrix at the reference geometry $\mathbf{x}_{0}$. As a consequence of Eq. (32) the differentiated integrals entering Eq. (31) are effectively expressed in the OMO basis. 
Equation (31) may therefore be expressed in the following form:

$$
\begin{aligned}
L_{\mathrm{MP} 2}^{(x)}= & 2 \sum_{i} \hat{h}_{i i}^{(x)}+\sum_{i j} \hat{L}_{i i j j}^{(x)} \\
& +\sum_{a i b j}\left(t_{i j}^{a b} \hat{L}_{a i b j}^{(x)}+\frac{1}{2} \bar{t}_{i j}^{a b} \hat{g}_{a i b j}^{(x)}\right) \\
& +\frac{1}{2} \sum_{a i b j c} \bar{t}_{i j}^{a b}\left(t_{i j}^{c b} \hat{F}_{a c}^{(x)}+t_{i j}^{a c} \hat{F}_{b c}^{(x)}\right) \\
& -\frac{1}{2} \sum_{a i b j k} \bar{t}_{i j}^{a b}\left(t_{k j}^{a b} \hat{F}_{k i}^{(x)}+t_{i k}^{a b} \hat{F}_{k j}^{(x)}\right) \\
& +2 \sum_{a i} \bar{\kappa}_{a i} \hat{F}_{a i}^{(x)}+h_{\mathrm{nuc}}^{(x)},
\end{aligned}
$$

where have used Eqs. (2), (3), and (11). For reasons of brevity we have omitted the $\left(\mathbf{x}_{0}\right)$ argument in Eq. (33) since all quantities are evaluated at $\mathbf{x}=\mathbf{x}_{0}$. This convention will be used throughout the paper unless otherwise indicated. Note that all differentiated integrals are written in the OMO basis and therefore contain both reorthonormalization contributions involving the differentiated overlap matrix and contributions involving differentiated UMO integrals. For example, the differentiated one-electron OMO integrals $\hat{h}_{p q}^{(x)}$ may be written as

$$
\begin{aligned}
\hat{h}_{p q}^{(x)}= & \left\langle\hat{\phi}_{p}\left|h_{1}\right| \hat{\phi}_{q}\right\rangle^{(x)} \\
= & -\frac{1}{2} \sum_{t}\left(\tilde{S}_{t p}^{(x)}\left\langle\phi_{t}\left|h_{1}\right| \phi_{q}\right\rangle+\tilde{S}_{t q}^{(x)}\left\langle\phi_{p}\left|h_{1}\right| \phi_{t}\right\rangle\right) \\
& +\left\langle\tilde{\phi}_{p}\left|h_{1}\right| \tilde{\phi}_{q}\right\rangle^{(x)} \\
\equiv & -\frac{1}{2} \sum_{t}\left(\tilde{S}_{t p}^{(x)} h_{t q}+\tilde{S}_{t q}^{(x)} h_{p t}\right)+\tilde{h}_{p q}^{(x)}
\end{aligned}
$$

where we have used Eqs. (16) and (20), and the fact that at $\mathbf{x}=\mathbf{x}_{0}$ the (non-differentiated) OMOs $\hat{\phi}$, the UMOs $\tilde{\phi}$, and the optimized MOs $\phi$ are identical, i.e., $\hat{\phi}\left(\mathbf{x}_{0}\right)=\tilde{\phi}\left(\mathbf{x}_{0}\right)$ $=\phi\left(\mathbf{x}_{0}\right)$, see Eqs. (15), (16), and (18).

It is convenient to collect the terms in Eq. (33) in different subgroups based on the differentiated integrals:

- Terms involving one-electron integrals $h^{(x)}$ are labeled $L_{1-\mathrm{el}}^{(x)}$.

- Terms involving the differentiated overlap matrix $S^{(x)}$ (reorthonormalization terms) are labeled $L_{\text {reort }}^{(x)}$.

- Terms involving differentiated two-electron integrals, corresponding to Coulomb or exchange transformations are written as $L_{\text {coulomb }}^{(x)}$ and $L_{\text {exchange }}^{(x)}$, respectively.

- Some of the terms involving differentiated twoelectron integrals cannot readily be expressed in terms of coulomb or exchange contributions. These terms will collectively be denoted $L_{\Theta}^{(x)}$.

- Finally, as already done above, the nuclear-nuclear repulsion term is denoted $h_{\text {nuc }}^{(x)}$.

When collecting the gradient terms as described above it is convenient to introduce some intermediates. The determination of these quantities using the DEC scheme is the central task when evaluating the DEC-MP2 molecular gradient in
Sec. III. The $\mathbf{X}, \mathbf{Y}$, and $\boldsymbol{\Theta}$ intermediates are given as

$$
\begin{gathered}
X_{i j}=\sum_{a b k} t_{k i}^{b a} \bar{t}_{k j}^{b a}=X_{j i}, \\
Y_{a b}=\sum_{c i j} t_{j i}^{c a} \bar{t}_{j i}^{c b}=Y_{b a}, \\
\Theta_{i j}^{a b}=4 t_{i j}^{a b}-2 t_{i j}^{b a}+\bar{t}_{i j}^{a b}=\Theta_{j i}^{b a} .
\end{gathered}
$$

The symmetry relations in Eqs. (35)-(37) follow trivially from the symmetry of the amplitudes $\left(t_{i j}^{a b}=t_{j i}^{b a}\right)$ and the relation between the amplitudes $t$ and multipliers $\bar{t}$ discussed in Sec. II E. Listing the orbitals with occupied orbitals preceding virtual ones, the MP2 correlation density matrix may be written as

$$
\boldsymbol{\rho}=\left(\begin{array}{cc}
-X_{i j} & \bar{\kappa}_{i a} \\
\bar{\kappa}_{a i} & Y_{a b}
\end{array}\right)=\boldsymbol{\rho}^{T},
$$

where $\bar{\kappa}_{i a}=\bar{\kappa}_{a i}$. We also introduce the $\boldsymbol{\Phi}$ matrix with the following sub-blocks,

$$
\begin{aligned}
\Phi_{i j}^{\mathrm{oo}} & =\sum_{a b k} \Theta_{k i}^{b a} g_{b k a j}, \\
\Phi_{i c}^{\mathrm{ov}} & =\sum_{a b k} \Theta_{k i}^{b a} g_{b k a c}, \\
\Phi_{a b}^{\mathrm{vv}} & =\sum_{c i j} \Theta_{j i}^{c a} g_{c j b i}, \\
\Phi_{a k}^{\mathrm{vo}} & =\sum_{c i j} \Theta_{j i}^{c a} g_{c j k i},
\end{aligned}
$$

where the "o" and " $v$ " superscripts refer to occupied and virtual subscript indices, respectively.

Using the partitioning of the gradient terms discussed above and the intermediates in Eqs. (35)-(42) the MP2 molecular gradient in the MO basis may be expressed as

$$
\begin{aligned}
L_{\mathrm{MP} 2}^{(x)}= & L_{1-\mathrm{el}}^{(x)}+L_{\mathrm{reort}}^{(x)}+L_{\text {coulomb }}^{(x)} \\
& +L_{\text {exchange }}^{(x)}+L_{\Theta}^{(x)}+h_{\mathrm{nuc}}^{(x)},
\end{aligned}
$$

where

$$
\begin{gathered}
L_{1 \text {-el }}^{(x)}=2 \sum_{i} \tilde{h}_{i i}^{(x)}+\sum_{p q} \rho_{p q} \tilde{h}_{p q}^{(x)}, \\
L_{\text {coulomb }}^{(x)}=2 \sum_{i j} \tilde{g}_{i i j j}^{(x)}+2 \sum_{i p q} \rho_{p q} \tilde{g}_{p q i i}^{(x)}, \\
L_{\text {exchange }}^{(x)}=-\sum_{i j} \tilde{g}_{i j j i}^{(x)}-\sum_{i p q} \rho_{p q} \tilde{g}_{p i i q}^{(x)}, \\
L_{\text {reort }}^{(x)}=-2 \sum_{i j} \tilde{S}_{i j}^{(x)} F_{j i}-\frac{1}{2} \sum_{p q} \tilde{S}_{p q}^{(x)} \Phi_{p q} \\
-\sum_{p q r} \tilde{S}_{r p}^{(x)} \rho_{p q} F_{q r} \\
-\sum_{i p q r} \tilde{S}_{r i}^{(x)} \rho_{p q}\left(2 \tilde{g}_{p q r i}-\tilde{g}_{p r i q}\right), \\
L_{\Theta}^{(x)}=\frac{1}{2} \sum_{i j a b} \Theta_{i j}^{a b} \tilde{g}_{a i b j}^{(x)} .
\end{gathered}
$$


The separation of the terms of the MP2 gradient in Eq. (43) is in accordance with the one of a conventional MP2 gradient calculation. ${ }^{44,46}$ For practical evaluation of the molecular gradient, it is convenient to transform Eq. (43) to the AO basis. Using the intermediates defined in Appendix A 1, the different terms of Eq. (43) may be written as

$$
\begin{gathered}
L_{1-\mathrm{el}}^{(x)}=\sum_{\mu \nu} h_{\mu \nu}^{(x)}(2 \mathbf{D}+\boldsymbol{\rho})_{\mu \nu}, \\
L_{\text {coulomb }}^{(x)}=2 \sum_{\mu \nu} J_{\mu \nu}^{(x)}(\mathbf{D})(\mathbf{D}+\boldsymbol{\rho})_{\mu \nu}, \\
L_{\text {exchange }}^{(x)}=-\sum_{\mu \nu} K_{\mu \nu}^{(x)}(\mathbf{D})(\mathbf{D}+\boldsymbol{\rho})_{\mu \nu}, \\
L_{\text {reort }}^{(x)}=-S_{\mu \nu}^{(x)} W_{\mu \nu}, \\
L_{\Theta}^{(x)}=\frac{1}{2} \sum_{i j} \sum_{\mu \nu \alpha \beta} \Theta_{\alpha \beta}^{(i j)} g_{\mu \alpha \beta \nu}^{(x)} C_{\mu \nu}^{(i j)} .
\end{gathered}
$$

\section{E. Multiplier equations}

By evaluating the variational criteria in Eq. (26) at $\mathbf{x}=\mathbf{x}_{0}$ the equation for the zeroth order $\bar{t}$ multipliers is found to be

$$
\begin{aligned}
2 L_{a i b j} & +\sum_{c}\left(\bar{t}_{i j}^{c b} F_{a c}+\bar{t}_{i j}^{a c} F_{b c}\right) \\
& -\sum_{k}\left(\bar{t}_{k j}^{a b} F_{k i}+\bar{t}_{i k}^{a b} F_{k j}\right)=0 .
\end{aligned}
$$

The multiplier equation in Eq. (54) has the same structure as the amplitude equation in Eq. (11) (evaluated at $\mathbf{x}=\mathbf{x}_{0}$ ). The only difference is that $g_{a i b j}$ in Eq. (11) is replaced by $2 L_{a i b j}$ $=4 g_{a i b j}-2 g_{a j b i}$ in Eq. (54). It thus follows that the $\bar{t}$ multipliers may be obtained directly from the $t$ amplitudes according to

$$
\bar{t}_{i j}^{a b}=4 t_{i j}^{a b}-2 t_{i j}^{b a}
$$

and the $\bar{t}$ equation in Eq. (54) therefore does not need to be solved in practice.

The equation for the zeroth order $\bar{\kappa}$ multipliers may be obtained from Eq. (28) evaluated at $\mathbf{x}=\mathbf{x}_{0}$. Using Eqs. (18) and (19) the derivative of a single molecular orbital $\phi_{p}(\mathbf{x})$ with respect to $\kappa_{d l}(\mathbf{x})$ (and evaluated at $\mathbf{x}=\mathbf{x}_{0}$ ) may be written as

$$
\left.\frac{\partial \phi_{p}(\mathbf{x})}{\partial \kappa_{d l}(\mathbf{x})}\right|_{\mathbf{x}=\mathbf{x}_{0}}=-\phi_{d}\left(\mathbf{x}_{0}\right) \delta_{p l}+\phi_{l}\left(\mathbf{x}_{0}\right) \delta_{p d} .
$$

By applying Eq. (56) and using the intermediates in Eqs. (35)-(42), Eq. (28) may - after some straightforward but lengthy algebra - be written as

$$
\begin{aligned}
& 2 \sum_{a} \bar{\kappa}_{a l} F_{a d}-2 \sum_{i} \bar{\kappa}_{d i} F_{l i} \\
& +2 \sum_{a i} \bar{\kappa}_{a i}\left(4 g_{a i d l}-g_{a d l i}-g_{a l d i}\right) \\
= & \Phi_{d l}^{\mathrm{vo}}-\Phi_{l d}^{\mathrm{ov}}+\sum_{i k} X_{i k}\left(4 g_{d l i k}-2 g_{d k i l}\right) \\
& -\sum_{a c} Y_{a c}\left(4 g_{d l a c}-2 g_{d c a l}\right) .
\end{aligned}
$$

In practice, the Coulomb and exchange transformations entering Eq. (57) are evaluated in the $\mathrm{AO}$ basis as discussed in Appendix A 2, and Eq. (57) is solved iteratively in the MO basis.

The expressions for the MP2 molecular gradient in Eq. (43) and the associated multiplier equations in Eqs. (55) and (57) are valid for any choice of optimized HF basis $\phi$. It has only been assumed that, at the reference geometry $\mathbf{x}_{0}$, the MOs are orthonormal (Eq. (13)) and the HF optimization condition (Eq. (14)) is satisfied. Some of the terms will simplify if the canonical basis is used. However, since the canonical orbitals are delocalized over the whole molecule such an approach is not suitable for an efficient description of local electron correlation effects. In contrast, using a set of local occupied and local virtual orbitals (in practice the HF orbitals were localized as described in Ref. 30), it is possible to apply the DEC scheme to evaluate the MP2 molecular gradient in a linear-scaling manner as we now describe.

\section{MP2 MOLECULAR GRADIENT USING THE DEC SCHEME}

In Sec. III A we summarize the evaluation of the MP2 Lagrangian correlation energy using the DEC Lagrangian partitioning scheme introduced in Ref. 29, where the MP2 correlation energy is determined in terms of small orbital fragment calculations. Then, in Secs. III B and III C, we use locality arguments to demonstrate that the MP2 gradient intermediates involving four-indexed amplitudes and integrals may also be evaluated within the DEC Lagrangian partitioning scheme. Finally, in Sec. III D we discuss how the trace of the MP2 correlation density provides a simple measure of the error of a DEC-MP2 calculation compared to a standard MP2 calculation.

\section{A. MP2 correlation energy using the Lagrangian partitioning scheme}

We first write the Lagrangian in Eq. (25) (evaluated at $\left.\mathbf{x}=\mathbf{x}_{0}\right)$ in terms of a HF component and a correlation component (keeping the $\mathbf{x}_{0}$ argument for clarity),

$$
\begin{gathered}
L_{\mathrm{MP} 2}\left(\mathbf{x}_{0}\right)=L_{\mathrm{HF}}\left(\mathbf{x}_{0}\right)+L_{\mathrm{corr}}\left(\mathbf{x}_{0}\right), \\
L_{\mathrm{HF}}\left(\mathbf{x}_{0}\right)=E_{\mathrm{HF}}\left(\mathbf{x}_{0}\right)+2 \sum_{a i} \bar{\kappa}_{a i}\left(\mathbf{x}_{0}\right) F_{a i}\left(\mathbf{x}_{0}\right) \\
=E_{\mathrm{HF}}\left(\mathbf{x}_{0}\right),
\end{gathered}
$$




$$
\begin{aligned}
L_{\mathrm{corr}}\left(\mathbf{x}_{0}\right)= & \sum_{a i b j} t_{i j}^{a b}\left(\mathbf{x}_{0}\right) L_{a i b j}\left(\mathbf{x}_{0}\right) \\
& +\frac{1}{2} \sum_{a i b j} \bar{t}_{i j}^{a b}\left(\mathbf{x}_{0}\right) \Omega_{i j}^{a b}\left(\mathbf{x}_{0}\right),
\end{aligned}
$$

where we have used Eq. (14) to obtain the second equality in Eq. (59). For a standard MP2 calculation we also have $\Omega_{i j}^{a b}\left(\mathbf{x}_{0}\right)=0$ if the MP2 amplitude equations have been solved exactly. However, in the DEC Lagrangian scheme, local amplitude equations are solved for local orbital spaces, and the full amplitude equation is never solved. It is therefore necessary to keep the second term of Eq. (60). ${ }^{29}$ We now describe the DEC scheme for evaluating $L_{\text {corr }}$ as a sum of fragment energies.

When a set of local orbitals is used, it is meaningful to assign each orbital $\phi_{r}$ to the atomic site $\mathrm{P}$ where it has the largest Löwdin charge. (Other assignment criteria may also be used but the Löwdin charge assignment is used for the results presented in this paper). In practice, orbitals originally assigned to hydrogen atoms are reassigned to the nearest heavy atom to obtain a more homogeneous orbital assignment. Having assigned all occupied and virtual orbitals to atomic sites the Lagrangian correlation energy may be evaluated as ${ }^{29}$

$$
L_{\mathrm{corr}}=\sum_{P} L_{\mathrm{P}}+\sum_{P>Q} \Delta L_{\mathrm{PQ}}
$$

where the summations run over all atomic sites, and where the atomic fragment energy $L_{\mathrm{P}}$ is given by

$$
\begin{aligned}
L_{\mathrm{P}}= & \sum_{i j \in \mathrm{P}} \sum_{a b}\left(t_{i j}^{a b} L_{a i b j}\right. \\
& \left.+\frac{1}{2} \sum_{c} \bar{t}_{i j}^{a b}\left(t_{i j}^{c b} F_{a c}+t_{i j}^{a c} F_{b c}\right)\right) \\
& +\sum_{a b \in \mathrm{P}} \sum_{i j}\left(\frac{1}{2} \bar{t}_{i j}^{a b} g_{a i b j}\right. \\
& \left.-\frac{1}{2} \sum_{k} \bar{t}_{i j}^{a b}\left(t_{k j}^{a b} F_{k i}+t_{i k}^{a b} F_{k j}\right)\right),
\end{aligned}
$$

while the pair interaction energy $\Delta L_{\mathrm{PQ}}$ is defined as

$$
\begin{aligned}
\Delta L_{\mathrm{PQ}}= & \left(\sum_{\substack{i \in \mathrm{P} \\
j \in \mathrm{Q}}}+\sum_{\substack{i \in \mathrm{Q} \\
j \in \mathrm{P}}}\right) \sum_{a b}\left(t_{i j}^{a b} L_{a i b j}\right. \\
& \left.+\frac{1}{2} \sum_{c} \bar{t}_{i j}^{a b}\left(t_{i j}^{c b} F_{a c}+t_{i j}^{a c} F_{b c}\right)\right) \\
& +\left(\sum_{\substack{a \in \mathrm{P} \\
b \in \mathrm{Q}}}+\sum_{\substack{a \in \mathrm{Q} \\
b \in \mathrm{P}}}\right) \sum_{i j}\left(\frac{1}{2} \bar{t}_{i j}^{a b} g_{a i b j}\right. \\
& \left.-\frac{1}{2} \sum_{k} \bar{t}_{i j}^{a b}\left(t_{k j}^{a b} F_{k i}+t_{i k} F_{k j}\right)\right) .
\end{aligned}
$$

No approximations have been made in Eq. (61). The Lagrangian correlation energy has simply been partitioned into atomic site and pair interaction contributions. Note that the occupied orbitals $\phi_{i}$ and $\phi_{j}$ in the two first terms in Eq. (62) are assigned to atomic site $\mathrm{P}$, while the virtual orbitals $\phi_{a}$ and $\phi_{b}$ are assigned to atomic site $\mathrm{P}$ in the last two terms of Eq. (62) (and likewise for Eq. (63)). The partitioning in Eqs. (62) and (63) thus allows for a uniform treatment of the occupied and virtual orbital spaces. This turns out to be important when evaluating the MP2 molecular gradient using the DEC scheme as will be demonstrated in Sec. III C.

When local virtual orbitals are used, many amplitudes and two-electron integrals will be (close to) zero. In particular, if occupied orbitals $\phi_{i}$ and $\phi_{j}$ are assigned to atomic site $\mathrm{P}$, integrals $g_{a i b j}$ will be non-vanishing only if virtual orbitals $\phi_{a}$ and $\phi_{b}$ are spatially close to P. We refer to the set of virtual orbitals "spatially close" to atomic site P (in a sense that will be defined below) as the virtual amplitude orbital space (AOS) for atomic site $\mathrm{P}$ and denote this space by the symbol $[\overline{\mathrm{P}}]$, i.e.,

$$
i j \in \mathrm{P}: g_{a i b j} \neq 0 \Rightarrow a b \in[\overline{\mathrm{P}}] \text {. }
$$

The locality of the amplitudes $t_{i j}^{a b}$ is closely related to the locality of the integrals via the amplitude equation in Eq. (11). In Ref. 28 a locality analysis is carried out which demonstrates that non-vanishing amplitudes $t_{i j}^{a b}(i j \in \mathrm{P})$ satisfy the following locality constraint:

$$
i j \in \mathrm{P}: t_{i j}^{a b} \neq 0 \Rightarrow a b \in[\overline{\mathrm{P}}] \text {. }
$$

More precisely, for $t_{i j}^{a b}$ with $i j \in \mathrm{P}$ to be nonzero, $\phi_{a}$ and $\phi_{b}$ must belong to $[\overline{\mathrm{P}}]$ itself or to buffer atoms surrounding $[\overline{\mathrm{P}}] .{ }^{28}$ However, in practice these buffer atoms are absorbed in $[\overline{\mathrm{P}}]$, so Eq. (65) is valid for all practical matters. Note that the locality restrictions in Eq. (65) also applies to the multipliers $\bar{t}$ by virtue of Eq. (55).

Similar arguments may be applied to the case where two virtual orbitals $\phi_{a}$ and $\phi_{b}$ are assigned to atomic site P. The integral $g_{a i b j}$ will now be non-vanishing only if $\phi_{i}$ and $\phi_{j}$ are spatially close to P. The occupied AOS containing the occupied orbitals spatially close to $\mathrm{P}$ will be denoted by the symbol $[\underline{\mathrm{P}}]$. We thus obtain the following analogues of Eqs. (64) and (65),

$$
a b \in \mathrm{P}: g_{a i b j} \neq 0 \Rightarrow i j \in[\underline{\mathrm{P}}]
$$

and

$$
a b \in \mathrm{P}: t_{i j}^{a b} \neq 0 \Rightarrow i j \in[\underline{\mathrm{P}}] \text {. }
$$

In addition to the locality restrictions listed above, we note that, for local orbitals, the Fock matrix elements will be local in the sense that $F_{i j}\left(F_{a b}\right)$ is non-vanishing only if orbitals $\phi_{i}$ and $\phi_{j}\left(\phi_{a}\right.$ and $\left.\phi_{b}\right)$ are spatially close. For a detailed locality analysis we refer to Ref. 28. 


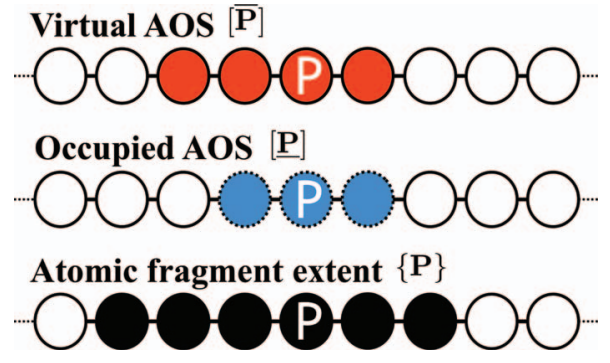

FIG. 1. Example of an atomic fragment P. Each circle represents an atom in a schematic linear molecule. White atoms are not considered in the calculation for atomic fragment P. MOs assigned to red/solid (blue/dotted) atoms are included in the virtual (occupied) amplitude orbital space (AOS) $[\overline{\mathrm{P}}]([\mathrm{P}])$. The amplitude equation is solved using the union of the occupied and virtual AOSs. Black atoms are included in the atomic fragment extent $\{\mathrm{P}\}$ (where the MOs in the AOS are expanded).

Using the locality constraints in Eqs. (64)-(67), Eq. (62) may be written as ${ }^{29}$

$$
\begin{aligned}
L_{\mathrm{P}}= & \sum_{i j \in \mathrm{P}} \sum_{a b \in[\overline{\mathrm{P}}]}\left(t_{i j}^{a b} L_{a i b j}\right. \\
& \left.+\frac{1}{2} \sum_{c \in[\overline{\mathrm{P}}]} \bar{t}_{i j}^{a b}\left(t_{i j}^{c b} F_{a c}+t_{i j}^{a c} F_{b c}\right)\right) \\
& +\sum_{a b \in \mathrm{P}} \sum_{i j \in[\underline{\mathrm{P}}]}\left(\frac{1}{2} \bar{t}_{i j}^{a b} g_{a i b j}\right. \\
& \left.-\frac{1}{2} \sum_{k \in[\underline{\mathrm{P}}]} \bar{t}_{i j}^{a b}\left(t_{k j}^{a b} F_{k i}+t_{i k}^{a b} F_{k j}\right)\right) .
\end{aligned}
$$

Figure 1 gives a schematic representation of the virtual AOS (red/solid) and the occupied AOS (blue/dotted) for an atomic fragment P. The amplitudes entering Eq. (68) are obtained by solving a local MP2 amplitude equation in the AOS (i.e., the union of the occupied AOS and the virtual AOS). In other words, only amplitudes $t_{i j}^{a b}$ where $i j \in[\underline{\mathrm{P}}]$ and $a b \in[\overline{\mathrm{P}}]$ enter the amplitude equation for fragment P. In summary, the locality restrictions in Eqs. (64)-(67) are used to evaluate the atomic fragment energy according to Eq. (68).

The two main error sources in DEC-MP2 calculations are the following:

(i) The summations in Eq. (68) are truncated and therefore (small) contributions from amplitudes outside the AOS are neglected.

(ii) The MP2 amplitude equation for fragment P is solved using only amplitudes inside the AOS ( $t_{i j}^{a b}$ for which $i j \in[\underline{\mathrm{P}}]$ and $a b \in[\overline{\mathrm{P}}]$ ). In this way (small) couplings between AOS amplitudes and amplitudes outside the AOS are neglected, and the AOS amplitudes will therefore differ slightly from the corresponding amplitudes determined in a full molecular calculation.

In a DEC calculation it is ensured that the total error in $L_{\mathrm{P}}$ - which is a combination of errors of type (i) and (ii) - is below a predefined precision denoted the fragment optimization threshold (FOT) ${ }^{27-29}$ This is achieved by first calculating $L_{\mathrm{P}}$ for some small AOS containing atoms neighbouring atom $P$.
The AOS is then expanded to include a second layer of atoms close to $P$, and $L_{\mathrm{P}}$ is then calculated again. If the difference between the two $L_{P}$ values is smaller than the FOT, the AOS is converged. If not, the AOS is expanded further, and $L_{\mathrm{P}}$ is recalculated in this new AOS. This black-box procedure is continued until convergence. The foundation for this procedure is the fact that, when local orbitals are used, the contributions to $L_{\mathrm{P}}$ from an atom $Q \neq P$ decays very rapidly with distance between $P$ and $Q .{ }^{28}$ In the orbital assignment it is of course possible that, for a given MO $\phi_{r}$, two atoms have roughly the same Löwdin charge, and $\phi_{r}$ is then assigned to one of these atoms, although it has an equally large Löwdin charge on the other atom. This is, however, not a practical problem as the fragment optimization procedure will automatically adapt the sizes of the local orbital spaces based on the specific orbital assignment. Thus, in general, the more orbitals are assigned to a given atom, the larger the AOS will be. A fragment optimization procedure for the (non-Lagrangian) CC energy is presented in Ref. 28. A fragment optimization procedure for the Lagrangian correlation energy $L_{\text {corr }}$ will be presented in a forthcoming paper.

We note that restrictions to the virtual excitation space similar to those in Eqs. (64) and (65) are used in other local CC approaches. ${ }^{3-6,12-14,17,18,22-26}$ The main difference between the various approaches lies in the determination of the local excitation spaces. In most methods the sizes of the virtual excitation spaces are chosen using some predefined threshold(s). In the limit where the threshold(s) goes to zero, the standard CC energy is recovered. However, in general it is difficult to estimate the error introduced in the CC energy by the various threshold approximations. In the DEC scheme the individual energy contributions $L_{\mathrm{P}}$ are directly targeted to ensure that they are determined to the threshold (the FOT), and in this way the calculated correlation energy is also determined to this threshold. In the DEC approach the local orbital spaces (both occupied and virtual) are thus adapted to each atom $P$ in a black box manner.

In general a local MO included in the AOS will have its largest MO coefficients on atoms in the AOS. However, there may be tail coefficients of very small - but non-negligible magnitudes outside the AOS. In other words, for a MO $\phi_{r}$ inside the AOS for fragment $\mathrm{P}$,

$$
\phi_{r}=\sum_{\mu} C_{\mu r} \chi_{\mu},
$$

the $\mu$ summation in principle runs over all atomic orbitals $\chi_{\mu}$ in the molecule, although the bulk of the orbital is located on the AOS atoms. To accurately describe small orbital tails outside the AOS, we effectively include an additional layer of "boundary atoms" outside the AOS. The total set of AOS atoms and boundary atoms for fragment $\mathrm{P}$ is denoted the atomic fragment extent $\{\mathrm{P}\}$ and is depicted by black circles in Figure 1. A molecular AOS orbital $\phi_{r}$ may now be accurately described in terms of an approximate orbital $\phi_{r}^{\prime}$ where the $\mu$ summation is restricted to the local atomic fragment extent,

$$
\phi_{r}^{\prime}=\sum_{\mu \in\{\mathrm{P}\}} C_{\mu r}^{\prime} \chi_{\mu} .
$$


The approximate MO coefficients $C_{\mu r}^{\prime}$ are determined such that $\phi_{r}^{\prime}$ resembles $\phi_{r}$ as closely as possible in a least-squares sense as proposed by Usvyat and Schütz. ${ }^{78}$ This is discussed in greater details in a DEC context in Sec. 6.1 of Ref. 28. The errors due to the approximation in Eq. (70) are particularly small for the central MOs in fragment $\mathrm{P}$ because the exact MO coefficients for these orbitals are basically vanishing outside the atomic fragment extent. The errors are somewhat larger for MOs assigned to boundary AOS atoms in Figure 1. However, the contribution to the fragment energy $L_{\mathrm{P}}$ from orbitals assigned to boundary AOS atoms are correspondingly smaller. ${ }^{28}$ Thus, the errors introduced due to the approximation in Eq. (70) are in general much smaller than the errors of type (i) and type (ii) discussed above. We note that in DEC fragment calculations we always use approximated orbitals $C^{\prime}$, for example when $\mathrm{AO}$ integrals are transformed to the MO basis.

By applying similar locality arguments as those in Eqs. (64)-(67) to the integrals and amplitudes entering the pair interaction energy in Eq. (75) we obtain the following locality restrictions: ${ }^{29}$

$$
\begin{aligned}
& i j \in \mathrm{P} \cup \mathrm{Q}: g_{a i b j} \neq 0 \Rightarrow a b \in[\overline{\mathrm{P}}] \cup[\overline{\mathrm{Q}}], \\
& i j \in \mathrm{P} \cup \mathrm{Q}: t_{i j}^{a b} \neq 0 \Rightarrow a b \in[\overline{\mathrm{P}}] \cup[\overline{\mathrm{Q}}], \\
& a b \in \mathrm{P} \cup \mathrm{Q}: g_{a i b j} \neq 0 \Rightarrow i j \in[\underline{\mathrm{P}}] \cup[\underline{\mathrm{Q}}], \\
& a b \in \mathrm{P} \cup \mathrm{Q}: t_{i j}^{a b} \neq 0 \Rightarrow i j \in[\underline{\mathrm{P}}] \cup[\underline{\mathrm{Q}}],
\end{aligned}
$$

where Eqs. (72) and (74) also hold for the $\bar{t}$ multipliers due to the relation in Eq. (55). Using Eqs. (71)-(74) the pair interaction energy may be approximated as ${ }^{29}$

$$
\begin{aligned}
& \Delta L_{\mathrm{PQ}}=\left(\sum_{\substack{i \in \mathrm{P} \\
j \in \mathrm{Q}}}+\sum_{\substack{i \in \mathrm{Q} \\
j \in \mathrm{P}}} \sum_{a b \in[\overline{\mathrm{P}}] \cup[\overline{\mathrm{Q}}]}\left(t^{a b} L_{a i b j}\right.\right. \\
& \left.+\frac{1}{2} \sum_{c \in[\overline{\mathrm{P}}] \cup[\overline{\mathrm{Q}}]} \bar{t}_{i j}^{a b}\left(t_{i j}^{c b} F_{a c}+t_{i j}^{a c} F_{b c}\right)\right) \\
& +\left(\sum_{\substack{a \in \mathrm{P} \\
b \in \mathrm{Q}}}+\sum_{\substack{a \in \mathrm{Q} \\
b \in \mathrm{P}}} \sum_{i j \in[\underline{\mathrm{P}]} \cup[\underline{\mathrm{Q}}]}\left(\frac{1}{2} \bar{t}^{a b} g_{a i b j}\right.\right. \\
& \left.-\frac{1}{2} \sum_{k \in[\underline{\mathrm{P}] \cup[\mathrm{Q}]}} \bar{t}_{i j}^{a b}\left(t_{k j}^{a b} F_{k i}+t_{i k} F_{k j}\right)\right) \text {. }
\end{aligned}
$$

The pair fragment PQ calculation thus employs unions of spaces from the atomic fragment calculations $\mathrm{P}$ and $\mathrm{Q}-$ i.e., the pair occupied AOS is $[\underline{P}] \cup[Q]$, the pair virtual AOS is $[\overline{\mathrm{P}}] \cup[\overline{\mathrm{Q}}]$, and the pair atomic fragment extent is $\{\mathrm{P}\} \cup\{\mathrm{Q}\}$. By calculating pair interaction energies using union of spaces from atomic fragment calculations, the error control of the atomic fragment energies $L_{\mathrm{P}}$ (in terms of the FOT) is implicitly imposed on the pair interaction energies $\Delta L_{\mathrm{PQ}}$, and therefore the error in the total correlation energy in Eq. (61) will also be proportional to the FOT. A higher precision of $L_{\text {corr }}$ (compared to a full molecular calculation) is thus obtained by lowering the FOT. Since the amplitude equation $\Omega_{i j}^{a b}$ enters directly in $L_{\text {corr }}$ in Eq. (60), a higher precision of $L_{\text {corr }}$ implies that $\Omega_{i j}^{a b}$ is evaluated to a higher precision, which is equivalent to a lower residual norm tolerance for the amplitude equation of the full molecular system. Lowering the FOT value in a DEC calculation is thus equivalent to lowering the residual norm tolerance for the amplitude equation in a full molecular calculation. The precision of the MP2 gradient is ultimately defined by the precision of the MP2 amplitudes, and a lower FOT value therefore implies a more accurate DEC-MP2 molecular gradient (compared to a full molecular calculation).

\section{B. Overview of the DEC-MP2 molecular gradient scheme}

In this section and Sec. III C we describe how the DEC scheme may be used to evaluate the MP2 gradient in Eq. (43) in a linear-scaling manner suitable for calculations on large molecular systems.

Disregarding $L_{\Theta}^{(x)}$ for the moment, the MP2 gradient expressions in Eqs. (49)-(52) only contains contributions of similar structure as the terms required to determine the HF gradient. In fact, if we set $\rho=\mathbf{0}, \mathbf{W}=2 \mathbf{D F D}$, and $L_{\Theta}^{(x)}=0$, then Eq. (43) reduces to the HF gradient. Thus, assuming that $\rho$ and $\mathbf{W}$ are available, Eqs. (49)-(52) may be evaluated for a large molecular system in a linear-scaling manner using similar techniques as for the HF gradient. ${ }^{71-73}$ The $\bar{\kappa}$ multiplier equation in Eq. (57) is effectively a HF response equation and it may therefore also be solved for large molecular systems using linear-scaling HF techniques. ${ }^{67-70}$ The main task in the DEC-MP2 gradient scheme is thus to perform all manipulations involving four-index quantities (integrals $g_{a i b j}$, amplitudes $t_{i j}^{a b}$, and multipliers $\left.\bar{t}_{i j}^{a b}\right)$ in terms of DEC fragment calculations.

The analysis of the MP2 molecular gradient is most easily carried out in the MO basis. A closer examination of the individual gradient contributions in Eqs. (44)-(48) shows that the intermediates involving four-index quantities are the $\mathbf{X}$ and $\mathbf{Y}$ matrices in Eqs. (35) and (36) (required for $\boldsymbol{\rho}$ and the right-hand side matrix of the $\bar{\kappa}$ equation in Eq. (57)), the $\boldsymbol{\Phi}$ matrix in Eqs. (39)-(42) (required for $\mathbf{W}$ and the right-hand side matrix in Eq. (57)), and the $L_{\Theta}^{(x)}$ gradient contribution in Eq. (48). In Sec. III C we use the locality constraints on the integrals and amplitudes discussed in Sec. III A to demonstrate how $\mathbf{X}, \mathbf{Y}, \boldsymbol{\Phi}$, and $L_{\Theta}^{(x)}$ may be evaluated by adding atomic fragment contributions $\mathbf{X}_{\mathrm{P}}, \mathbf{Y}_{\mathrm{P}}, \boldsymbol{\Phi}_{\mathrm{P}}$, and $L_{\Theta, \mathrm{P}}^{(x)}$ and pair fragment contributions $\Delta \mathbf{X}_{\mathrm{PQ}}, \Delta \mathbf{Y}_{\mathrm{PQ}}, \Delta \boldsymbol{\Phi}_{\mathrm{PQ}}$, and $\Delta L_{\Theta, \mathrm{PQ}}^{(x)}$. This is completely analogous to the evaluation of $L_{\text {corr }}$ in Eq. (61) in terms of atomic fragment energies $L_{\mathrm{P}}$ and pair interaction energies $\Delta L_{\mathrm{PQ}}$. Before going into detail with $\mathbf{X}, \mathbf{Y}, \boldsymbol{\Phi}$, and $L_{\Theta}^{(x)}$, it is appropriate to give an overview of the different steps involved in the evaluation of the MP2 gradient using the DEC scheme:

- The HF energy calculation is carried out and a set of local and orthogonal HF orbitals is constructed.

- A series of small independent atomic fragment calculations for each atomic site $\mathrm{P}$ is carried out to 
determine the atomic fragment contributions $\mathbf{X}_{\mathrm{P}}, \mathbf{Y}_{\mathrm{P}}$, $\boldsymbol{\Phi}_{\mathrm{P}}$, and $L_{\Theta, \mathrm{P}}^{(x)}$. (to be detailed in Sec. III C).

- The independent pair fragment calculations PQ are carried out to determine the pair fragment contributions $\Delta \mathbf{X}_{\mathrm{PQ}}, \Delta \mathbf{Y}_{\mathrm{PQ}}, \Delta \boldsymbol{\Phi}_{\mathrm{PQ}}$, and $\Delta L_{\Theta, \mathrm{PQ}}^{(x)}$ (to be detailed in Sec. III C).

- The full molecular intermediates $\mathbf{X}, \mathbf{Y}$, and $\boldsymbol{\Phi}$ are calculated by adding the fragment contributions (to be detailed in Sec. III C).

- The $\bar{\kappa}$ orbital-rotation multiplier equation in Eq. (57) is solved for the full molecule using $\mathbf{X}, \mathbf{Y}$, and $\boldsymbol{\Phi}$ to determine the right-hand side of the equation.

- The $\rho$ (Eq. (38)) and W (Eq. (A4)) matrices are determined using $\mathbf{X}, \mathbf{Y}$, and $\boldsymbol{\Phi}$.

- The molecular gradient in Eq. (43) is calculated from the individual gradient contributions in Eqs. (49)-(53), where the $L_{\Theta}^{(x)}$ contribution is calculated by adding the $L_{\Theta, \mathrm{P}}^{(x)}$ and $\Delta L_{\Theta, \mathrm{PQ}}^{(x)}$ fragment contributions (to be detailed in Sec. III C).

An overview of the main steps is given in Figure 2 where the yellow/dotted (blue/solid) boxes denote calculations involving orbital fragments (the full molecule). The MP2 gradient scheme depicted in Figure 2 thus treats all four-index integrals and amplitudes (yellow/dotted boxes) at the fragment level, while HF-like manipulations (including Coulomb and exchange transformations and contractions of two-index matrices) are carried out for the full molecule (blue/solid boxes). Thus, four-indexed quantities are never constructed for the full molecular system.

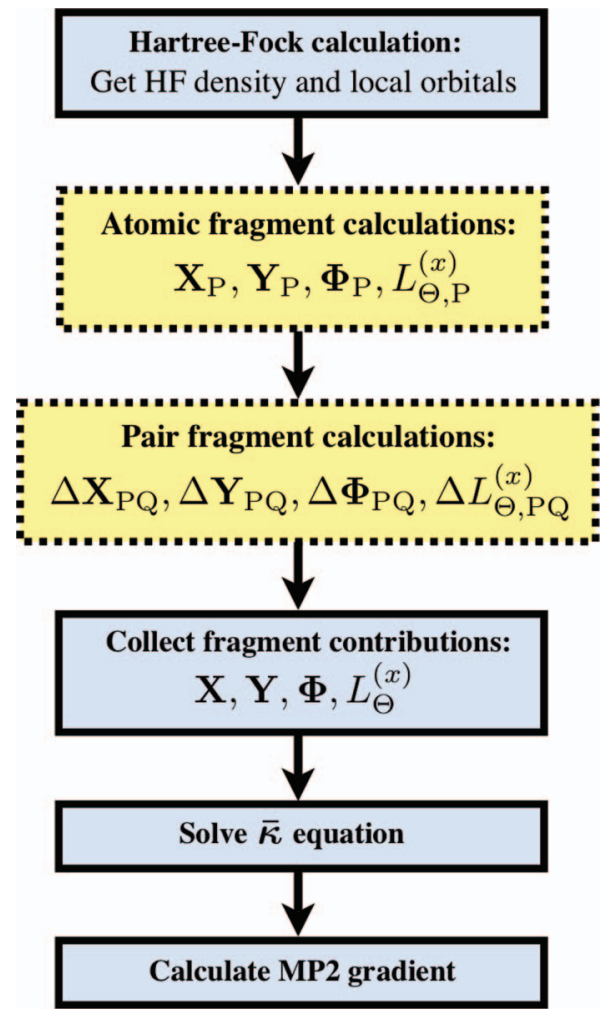

FIG. 2. Overview of the DEC-MP2 gradient scheme. Yellow/dotted boxes are fragment calculations, while blue/solid boxes are calculations involving the full molecule.
Let us conclude this section by commenting on the computational scaling of the DEC-MP2 gradient scheme in Figure 2. The number of atomic fragment calculations to consider scales linearly with system size, while the number of pair fragment calculations formally scales quadratically with system size. However, pair fragments describe dispersion effects ${ }^{28,29}$ which decay with the inverse pair distance to the sixth power for the energy and to the seventh power for the gradient. Pairs where the corresponding atomic sites are well separated in space may therefore be omitted from the calculation without affecting the precision (this is supported by numerical results in Sec. IV B). Thus, the total number of fragments to consider scales linearly with system size for large systems. Furthermore, the fragment sizes (the number of orbitals in the AOS) are roughly independent of the molecular size, ${ }^{28,29}$ and the computational cost for the correlated part of the DEC-MP2 gradient calculation (yellow/dotted boxes in Figure 2) therefore scales linearly with system size. In summary, assuming that the HF-like manipulations (blue/solid boxes in Figure 2) are carried out in a linearscaling manner, ${ }^{67-73}$ the computational time for the entire MP2 gradient scheme depicted in Figure 2 is linear-scaling with system size for large molecular systems. The fragment calculations (yellow/dotted boxes in Figure 2) are furthermore independent and thus embarrassingly parallel.

In the current implementation the HF-like parts of the DEC-MP2 gradient calculation are implemented using linearscaling techniques. ${ }^{67-73}$ However, at present the HF-like parts are not massively parallel as is the case for the correlated part of the calculation, and it therefore constitutes a bottleneck when carrying out large-scale DEC calculations on a super computer. Work is currently being carried out in our laboratory to remove this bottleneck by introducing efficient parallelization of the Coulomb and exchange transformations. It should also be noted that at present the matrix multiplications are formally cubically scaling, but this does not constitute a bottleneck in practice. The scaling of matrix multiplications may be reduced to linear for large systems if sparse matrix algebra is employed.

\section{DEC evaluation of MP2 gradient intermediates}

The DEC energy scheme involves atomic fragment energies and pair interaction energies. In the two first terms of the atomic fragment energy $L_{\mathrm{P}}$ in Eq. (62) (pair interaction energy $\Delta L_{\mathrm{PQ}}$ in Eq. (63)) two occupied orbitals $i, j$ are assigned to a specific atomic site $P$ (two atomic sites $P$ or $Q)$, while the virtual summations run freely. In the DEC terminology such terms are partitioned according to the occupied partitioning scheme. In the last two terms in Eq. (62) (Eq. (63)) two virtual indices $a, b$ are assigned to a specific atomic site $P$ (two atomic sites $P$ or $Q$ ), while the occupied summations run freely. Such terms are partitioned according to the virtual partitioning scheme. In Eqs. (68) and (75) locality approximations are introduced to restrict the virtual (occupied) summations in the occupied (virtual) partitioning terms to allow the fragment energies to be evaluated using small local orbital spaces. Since the DEC energy scheme inherently 
involves terms applying either the occupied or the virtual partitioning scheme, the goal of this section is to demonstrate that the gradient intermediates $\mathbf{X}, \mathbf{Y}, \boldsymbol{\Phi}$, and $L_{\Theta}^{(x)}$ may also be evaluated using either the occupied or the virtual partitioning scheme. This procedure is twofold. First, without summation restrictions the gradient intermediates need to be formulated in terms of atomic and pair interaction contributions using the either occupied or the virtual partitioning scheme. Second, we use the locality restrictions in Sec. III A to demonstrate that the summations entering the gradient intermediates may be restricted to the same local orbital spaces used for the fragment energy evaluations. In this way the DEC energy scheme is naturally extended to the determination of gradient intermediates (yellow/dotted boxes in Figure 2).

Let us first consider the $\mathbf{Y}$ matrix in Eq. (36). This expression involves contractions over two occupied indices $i$ and $j$ but only a summation over a single virtual index. For this reason, an element $Y_{a b}$ is naturally evaluated using the occupied partitioning scheme, i.e.,

$$
Y_{a b}=\sum_{\mathrm{P}}\left(Y_{\mathrm{P}}\right)_{a b}+\sum_{P>Q}\left(\Delta Y_{\mathrm{PQ}}\right)_{a b},
$$

where

$$
\begin{gathered}
\left(Y_{\mathrm{P}}\right)_{a b}=\sum_{i j \in \mathrm{P}} \sum_{c} t_{j i}^{c a} \bar{t}_{j i}^{c b}, \\
\left(\Delta Y_{\mathrm{PQ}}\right)_{a b}=\left(\sum_{\substack{i \in \mathrm{P} \\
j \in \mathrm{Q}}}+\sum_{\substack{i \in \mathrm{Q} \\
j \in \mathrm{P}}}\right) \sum_{c} t_{j i}^{c a} \bar{t}_{j i}^{c b} .
\end{gathered}
$$

No approximations have been made in Eq. (76). The full orbital summations have simply been partitioned into atomic $\mathrm{P}$ and pair interaction PQ contributions, and we have not gained any savings so far. However, for local orbitals the amplitudes in Eq. (77) satisfy the locality constraint given in Eq. (65). By virtue of Eq. (55) the multipliers satisfy the same conditions. Thus, if $i j \in \mathrm{P}$, then only $a b c$ indices for which $a b c \in[\overline{\mathrm{P}}]$ need to be considered in Eq. (77). It is thus sufficient to consider an atomic fragment matrix $\mathbf{Y}_{\mathrm{P}}$ of dimension $\left(N_{[\overline{\mathrm{P}}]} \times N_{[\overline{\mathrm{P}}]}\right)$, where $N_{[\overline{\mathrm{P}}]}$ is the number of orbitals in the virtual AOS for atomic fragment $P$ (orbitals assigned to $\mathrm{red} / \mathrm{solid}$ atoms in Figure 1):

$$
\left(Y_{\mathrm{P}}\right)_{a b}=\sum_{i j \in \mathrm{P}} \sum_{c \in[\overline{\mathrm{P}}]} t_{j i}^{c a} \bar{t}_{j i}^{c b} \quad(a b \in[\overline{\mathrm{P}}]) .
$$

Similarly, using Eq. (72) we may restrict the virtual indices of the $\Delta \mathbf{Y}_{\mathrm{PQ}}$ matrix and define a pair fragment matrix of dimensions $\left(N_{[\overline{\mathrm{P}}] \cup[\overline{\mathrm{Q}}]} \times N_{[\overline{\mathrm{P}}] \cup[\overline{\mathrm{Q}}]}\right)$,

$$
\begin{aligned}
\left(\Delta Y_{\mathrm{PQ}}\right)_{a b}= & \left(\sum_{\substack{i \in \mathrm{P} \\
j \in \mathrm{Q}}}+\sum_{\substack{i \in \mathrm{Q} \\
j \in \mathrm{P}}} \sum_{c \in[\overline{\mathrm{P}}] \cup[\overline{\mathrm{Q}}]} t_{j i}^{c a} \bar{t}_{j i}^{c b},\right. \\
& (a b \in[\overline{\mathrm{P}}] \cup[\overline{\mathrm{Q}}]) .
\end{aligned}
$$

By carrying out a series of fragment calculations and adding the fragment matrices in Eqs. (79) and (80) according to Eq. (76) (keeping track of the indices and recalling that the fragment matrices have different dimensions) we thus obtain an accurate representation of the total $\mathbf{Y}$ matrix.

The determination of $X_{i j}$ using Eq. (35) involves a summation over two virtual indices $a b$ and only one occupied index $k$. It is therefore necessary to employ the virtual partitioning scheme for the $\mathbf{X}$ matrix,

$$
X_{i j}=\sum_{\mathrm{P}}\left(X_{\mathrm{P}}\right)_{i j}+\sum_{P>Q}\left(\Delta X_{\mathrm{PQ}}\right)_{i j},
$$

where

$$
\begin{gathered}
\left(X_{\mathrm{P}}\right)_{i j}=\sum_{a b \in \mathrm{P}} \sum_{k} t_{k i}^{b a} \bar{t}_{k j}^{b a}, \\
\left(\Delta X_{\mathrm{PQ}}\right)_{i j}=\left(\sum_{\substack{a \in \mathrm{P} \\
b \in \mathrm{Q}}}+\sum_{\substack{a \in \mathrm{Q} \\
b \in \mathrm{P}}}\right) \sum_{k} t_{k i}^{b a} \bar{t}_{k j}^{b a} .
\end{gathered}
$$

Using Eqs. (67) and (74) (and equivalent equations for the $\bar{t}$ multipliers) we may now define an atomic fragment matrix $X_{a b}^{\mathrm{P}}$ of dimensions $\left(N_{[\mathrm{P}]} \times N_{[\mathrm{P}]}\right)$ and a pair fragment matrix $\Delta X_{a b}^{\mathrm{P}}$ of dimensions $\left(N_{[\underline{\mathrm{P}}] \cup[\underline{\mathrm{Q}}]} \times N_{[\underline{\mathrm{P}}] \cup[\underline{\mathrm{Q}}]}\right)$ according to

$$
\left(X_{\mathrm{P}}\right)_{i j}=\sum_{a b \in \mathrm{P}} \sum_{k \in[\underline{\mathrm{P}}]} t_{k i}^{b a} \bar{t}_{k j}^{b a}, \quad(i j \in[\underline{\mathrm{P}}])
$$

and

$$
\begin{aligned}
\left(\Delta X_{\mathrm{PQ}}\right)_{i j}= & \left(\sum_{\substack{a \in \mathrm{P} \\
b \in \mathrm{Q}}}+\sum_{\substack{a \in \mathrm{Q} \\
b \in \mathrm{P}}} \sum_{k \in[\underline{\mathrm{P}}] \cup[\underline{\mathrm{Q}}]} t_{k i}^{b a} \bar{t}_{k j}^{b a},\right. \\
& (i j \in[\underline{\mathrm{P}]} \cup[\underline{\mathrm{Q}}]) .
\end{aligned}
$$

The $\mathbf{Y}$ matrix in Eq. (76) is thus evaluated using the occupied partitioning scheme, while the $\mathbf{X}$ matrix in Eq. (81) is evaluated using the virtual partitioning scheme. Note that there are no other possibilities within the DEC scheme. Since the occupied (virtual) partitioning scheme requires a summation over two occupied (virtual) indices, the inherent structures of the $\mathbf{Y}$ and $\mathbf{X}$ matrices tie them to the occupied and virtual partitioning schemes, respectively. Only when the equations for the gradient intermediates are adapted to the DEC scheme can the locality approximations in Sec. III A be rigorously employed. In the DEC-MP2 gradient scheme it is therefore crucial to invoke both the occupied and the virtual partitioning schemes.

The evaluation of $\boldsymbol{\Phi}$ and $L_{\Theta}^{(x)}$ follow the same philosophy used for $\mathbf{Y}$ and $\mathbf{X}$ above. For completeness we list all the defining equations here. Consider first the $\boldsymbol{\Phi}$ matrix in Eqs. (39)-(42). It follows from Eqs. (37), (55), (65), (67), (72), and (74) that an element $\Theta_{i j}^{a b}$ therefore obeys the same locality restrictions as the amplitudes,

$$
\begin{aligned}
& i j \in \mathrm{P}: \quad \Theta_{i j}^{a b} \neq 0 \Rightarrow a b \in[\overline{\mathrm{P}}], \\
& a b \in \mathrm{P}: \quad \Theta_{i j}^{a b} \neq 0 \Rightarrow i j \in[\underline{\mathrm{P}}],
\end{aligned}
$$

$$
\begin{aligned}
& i j \in \mathrm{P} \cup \mathrm{Q}: \quad \Theta_{i j}^{a b} \neq 0 \Rightarrow a b \in[\overline{\mathrm{P}}] \cup[\overline{\mathrm{Q}}], \\
& a b \in \mathrm{P} \cup \mathrm{Q}: \quad \Theta_{i j}^{a b} \neq 0 \Rightarrow i j \in[\underline{\mathrm{P}}] \cup[\underline{\mathrm{Q}}] .
\end{aligned}
$$


Using Eqs. (86) and (88) and the locality restrictions for the integrals in Eqs. (64) and (71), $\Phi_{a b}^{\mathrm{vv}}$ may be evaluated using the occupied partitioning scheme,

$$
\begin{gathered}
\Phi_{a b}^{\mathrm{vv}}=\sum_{\mathrm{P}}\left(\Phi_{\mathrm{P}}^{\mathrm{vv}}\right)_{a b}+\sum_{P>Q}\left(\Delta \Phi_{\mathrm{PQ}}^{\mathrm{vv}}\right)_{a b}, \\
\left(\Phi_{\mathrm{P}}^{\mathrm{vv}}\right)_{a b}=\sum_{i j \in \mathrm{P}} \sum_{c \in[\overline{\mathrm{P}}]} \Theta_{j i}^{c a} g_{c j b i}, \quad(a b \in[\overline{\mathrm{P}}]), \\
\left(\Delta \Phi_{\mathrm{PQ}}^{\mathrm{vv}}\right)_{a b}=\left(\sum_{\substack{i \in \mathrm{P} \\
j \in \mathrm{Q}}}+\sum_{\substack{i \in \mathrm{Q} \\
j \in \mathrm{P}}}\right) \sum_{c \in[\overline{\mathrm{P}}] \cup[\overline{\mathrm{Q}}]} \Theta_{j i}^{c a} g_{c j b i}, \\
(a b \in[\overline{\mathrm{P}}] \cup[\overline{\mathrm{Q}}]) .
\end{gathered}
$$

Similarly, using the locality restrictions in Eqs. (66), (73), (87), and (89), $\Phi_{i j}^{\text {oo }}$ is naturally calculated using the virtual partitioning scheme,

$$
\begin{aligned}
\Phi_{i j}^{\mathrm{oo}}= & \sum_{\mathrm{P}}\left(\Phi_{\mathrm{P}}^{\mathrm{oo}}\right)_{i j}+\sum_{P>Q}\left(\Delta \Phi_{\mathrm{PQ}}^{\mathrm{oo}}\right)_{i j}, \\
\left(\Phi_{\mathrm{P}}^{\mathrm{oo}}\right)_{i j}= & \sum_{a b \in \mathrm{P}} \sum_{k \in[\underline{\mathrm{P}}]} \Theta_{k i}^{b a} g_{b k a j}, \quad(i j \in[\underline{\mathrm{P}}]), \\
\left(\Delta \Phi_{\mathrm{PQ}}^{\mathrm{oo}}\right)_{i j}= & \left(\sum_{\substack{a \in \mathrm{P} \\
b \in \mathrm{Q}}}+\sum_{\substack{a \in \mathrm{Q} \\
b \in \mathrm{P}}}\right) \sum_{k \in[\underline{\mathrm{P}}] \cup[\underline{\mathrm{Q}}]} \Theta_{k i}^{b a} g_{b k a j}, \\
& (i j \in[\underline{\mathrm{P}}] \cup[\underline{\mathrm{Q}}]) .
\end{aligned}
$$

The intermediates considered so far involve integrals with two occupied and two virtual indices, while the evaluation of $\Phi_{a k}^{\mathrm{vo}}\left(\Phi_{i c}^{\mathrm{ov}}\right)$ requires integrals with three occupied and one virtual (three virtual and one occupied) indices. We thus need to consider slightly modified locality restrictions for these integrals. To develop such modified locality restrictions consider first the locality condition in Eq. (64) with the indices renamed,

$$
i j \in \mathrm{P}: g_{c j b i} \neq 0 \Rightarrow c \in[\overline{\mathrm{P}}], \quad b \in[\overline{\mathrm{P}}] .
$$

Next we note that the integral $g_{c j k i}$ used for determining the $\boldsymbol{\Phi}^{\mathrm{vo}}$ matrix in Eq. (42) is the same as the one in Eq. (96) if the occupied index $k$ in $g_{c j k i}$ were replaced by a virtual index $b$. Since $[\overline{\mathrm{P}}]([\underline{\mathrm{P}}])$ denotes the set of virtual (occupied) orbitals spatially close to atomic site $\mathrm{P}$, we may therefore replace the locality requirement $b \in[\overline{\mathrm{P}}]$ for the $g_{c j b i}$ integral by the analogous requirement $k \in[\underline{\mathrm{P}}]$ for the $g_{c j k i}$ integral,

$$
i j \in \mathrm{P}: g_{c j k i} \neq 0 \Rightarrow c \in[\overline{\mathrm{P}}], \quad k \in[\underline{\mathrm{P}}] .
$$

Similar locality arguments may be applied to integrals where $\phi_{i}$ and $\phi_{j}$ are located on either center $\mathrm{P}$ or center $\mathrm{Q}$ to give

$$
i j \in \mathrm{P} \cup \mathrm{Q}: g_{c j k i} \neq 0 \Rightarrow c \in[\overline{\mathrm{P}}] \cup[\overline{\mathrm{Q}}], \quad k \in[\underline{\mathrm{P}}] \cup[\underline{\mathrm{Q}}] .
$$

Applying the locality restrictions in Eqs. (86), (88), (97), and (98), the $\boldsymbol{\Phi}^{\mathrm{vo}}$ matrix in Eq. (42) may be calculated using the occupied partitioning scheme,

$$
\Phi_{a k}^{\mathrm{vo}}=\sum_{\mathrm{P}}\left(\Phi_{\mathrm{P}}^{\mathrm{vo}}\right)_{a k}+\sum_{P>Q}\left(\Delta \Phi_{\mathrm{PQ}}^{\mathrm{vo}}\right)_{a k}
$$

$$
\begin{gathered}
\left(\Phi_{\mathrm{P}}^{\mathrm{vo}}\right)_{a k}=\sum_{i j \in \mathrm{P}} \sum_{c \in[\overline{\mathrm{P}}]} \Theta_{j i}^{c a} g_{c j k i}, \\
(a \in[\overline{\mathrm{P}}], k \in[\underline{\mathrm{P}}]), \\
\left(\Delta \Phi_{\mathrm{PQ}}^{\mathrm{vo}}\right)_{a k}=\left(\sum_{\substack{i \in \mathrm{P} \\
j \in \mathrm{Q}}}+\sum_{\substack{i \in \mathrm{Q} \\
j \in \mathrm{P}}}\right) \sum_{c \in[\overline{\mathrm{P}}] \cup[\overline{\mathrm{Q}}]} \Theta_{j i}^{c a} g_{c j k i}, \\
(a \in[\overline{\mathrm{P}}] \cup[\overline{\mathrm{Q}}], k \in[\underline{\mathrm{P}}] \cup[\underline{\mathrm{Q}}]) .
\end{gathered}
$$

For the $\Phi^{\text {ov }}$ matrix integrals $g_{b k a c}$ with three virtual indices are required. Using similar arguments as above, these integrals obey the following locality restrictions:

$$
a b \in \mathrm{P}: g_{b k a c} \neq 0 \Rightarrow k \in[\underline{\mathrm{P}}], \quad c \in[\overline{\mathrm{P}}],
$$

$a b \in \mathrm{P} \cup \mathrm{Q}: g_{b k a c} \neq 0 \Rightarrow k \in[\underline{\mathrm{P}}] \cup[\underline{\mathrm{Q}}], \quad c \in[\overline{\mathrm{P}}] \cup[\overline{\mathrm{Q}}]$.

Applying the locality restrictions in Eqs. (87), (89), (102), and (103) the $\boldsymbol{\Phi}^{\text {ov }}$ matrix may be evaluated according to the virtual partitioning scheme,

$$
\begin{gathered}
\Phi_{i c}^{\mathrm{ov}}=\sum_{\mathrm{P}}\left(\Phi_{\mathrm{P}}^{\mathrm{ov}}\right)_{i c}+\sum_{P>Q}\left(\Delta \Phi_{\mathrm{PQ}}^{\mathrm{ov}}\right)_{i c}, \\
\left(\Phi_{\mathrm{P}}^{\mathrm{ov}}\right)_{i c}=\sum_{a b \in \mathrm{P}} \sum_{k \in[\underline{\mathrm{p}}]} \Theta_{k i}^{b a} g_{b k a c}, \\
(i \in[\underline{\mathrm{P}}], c \in[\overline{\mathrm{P}}]), \\
\left(\Delta \Phi_{\mathrm{PQ}}^{\mathrm{ov}}\right)_{i c}=\left(\sum_{\substack{a \in \mathrm{P} \\
b \in \mathrm{Q}}}+\sum_{\substack{a \in \mathrm{Q} \\
b \in \mathrm{P}}}\right) \sum_{k \in[\underline{\mathrm{P}}] \cup[\underline{\mathrm{Q}}]} \Theta_{k i}^{b a} g_{b k a c}, \\
(i \in[\underline{\mathrm{P}}] \cup[\underline{\mathrm{Q}}], c \in[\overline{\mathrm{P}}] \cup[\overline{\mathrm{Q}}]) .
\end{gathered}
$$

In a full molecular calculation integrals with three virtual indices are difficult to handle memory-wise. However, since indices $a$ and $b$ in Eq. (105) (Eq. (106)) are restricted to a single atomic site (two atomic sites) and since the remaining indices are restricted to local orbital spaces, the integrals with three virtual indices in Eq. (105) (Eq. (106)) can be kept in memory. The DEC based evaluation of the $\boldsymbol{\Phi}^{\text {ov }}$ thus removes a major bottleneck in standard MP2 gradient calculations.

Let us finally describe how the $L_{\Theta}^{(x)}$ contribution to the molecular gradient in Eq. (48) may be evaluated in terms of fragment calculations. The determination of $L_{\Theta}^{(x)}$ requires differentiated two-electron integrals $\tilde{g}_{a i b j}^{(x)}$, and the locality of these integrals have not been considered so far. Let us consider differentiation with respect to a specific nuclear coordinate $y$ for an atom $Y$. Differentiation of one of the charge distributions entering $\tilde{g}_{a i b j}^{(y)}$ yields

$$
\left(\tilde{\phi}_{a} \tilde{\phi}_{i}\right)^{(y)}=\tilde{\phi}_{a}^{(y)} \phi_{i}+\phi_{a} \tilde{\phi}_{i}^{(y)} .
$$

Only AOs centered on atom $Y$ contribute to $\tilde{\phi}_{a}^{(y)}$ and $\tilde{\phi}_{i}^{(y)}$, and $\left(\tilde{\phi}_{a} \tilde{\phi}_{i}\right)^{(y)}$ will therefore have non-vanishing contributions only when the local orbitals $\tilde{\phi}_{i}$ and $\tilde{\phi}_{a}$ are spatially close to atom $Y$. This enforces an even stronger locality restriction on the 
$\tilde{g}_{a i b j}^{(y)}$ integrals than is the case for the non-differentiated $g_{a i b j}$ integrals. Thus, in general we are on the safe side if we assume that the differentiated two-electron integrals $\tilde{g}_{a i b j}^{(x)}$ have the same locality as the non-differentiated two-electron integrals in Eqs. (64) and (71). Using this assumption and the locality restrictions in Eqs. (86) and (88) the $L_{\Theta}^{(x)}$ contribution to the molecular gradient in Eq. (48) may be evaluated using the occupied partitioning scheme,

$$
\begin{gathered}
L_{\Theta}^{(x)}=\sum_{\mathrm{P}} L_{\Theta, \mathrm{P}}^{(x)}+\sum_{\mathrm{P}>\mathrm{Q}} \Delta L_{\Theta, \mathrm{PQ}}^{(x)}, \\
L_{\Theta, \mathrm{P}}^{(x)}=\frac{1}{2} \sum_{i j \in \mathrm{P}} \sum_{a b \in[\overline{\mathrm{P}}]} \Theta_{i j}^{a b} \tilde{g}_{a i b j}^{(x)}, \\
\Delta L_{\Theta, \mathrm{PQ}}^{(x)}=\frac{1}{2}\left(\sum_{\substack{i \in \mathrm{P} \\
j \in \mathrm{Q}}}+\sum_{\substack{i \in \mathrm{Q} \\
j \in \mathrm{P}}} \sum_{a b \in[\overline{\mathrm{P}}] \cup[\overline{\mathrm{Q}}]} \Theta_{i j}^{a b} \tilde{g}_{a i b j}^{(x)} .\right.
\end{gathered}
$$

In practice the integrals entering $L_{\Theta}^{(x)}$ are calculated in the $\mathrm{AO}$ basis. In a full calculation this corresponds to Eq. (53). In fragment calculations $L_{\Theta, \mathrm{P}}^{(x)}\left(\Delta L_{\Theta, \mathrm{PQ}}^{(x)}\right)$ is also evaluated in the AO basis using approximated molecular orbitals with fitted MO coefficients only on atoms in the atomic fragment extent $\{P\}(\{P\} \cup\{Q\})$, see Eq. (70) and the accompanying discussion. In the AO basis $L_{\Theta, \mathrm{P}}^{(x)}$ and $\Delta L_{\Theta, \mathrm{PQ}}^{(x)}$ may thus be evaluated as

$$
\begin{gathered}
L_{\Theta, \mathrm{P}}^{(x)}=\frac{1}{2} \sum_{i j \in \mathrm{P}} \sum_{\mu \nu \alpha \beta \in\{\mathrm{P}\}} \Theta_{\alpha \beta}^{(i j)} g_{\mu \alpha \beta \nu}^{(x)} C_{\mu \nu}^{(i j)}, \\
\Delta L_{\Theta, \mathrm{PQ}}^{(x)}=\frac{1}{2}\left(\sum_{\substack{i \in \mathrm{P} \\
j \in \mathrm{Q}}}+\sum_{\substack{i \in \mathrm{Q} \\
j \in \mathrm{P}}} \sum_{\mu \nu \alpha \beta \in\{\mathrm{P}\} \cup\{\mathrm{Q}\}} \Theta_{\alpha \beta}^{(i j)} g_{\mu \alpha \beta \nu}^{(x)} C_{\mu \nu}^{(i j)},\right.
\end{gathered}
$$

where $C_{\mu \nu}^{(i j)}$ and $\Theta_{\alpha \beta}^{(i j)}$ given in Eqs. (A10) and (A11) are now determined in terms of fitted MO coefficients,

$$
\begin{gathered}
C_{\mu \nu}^{(i j)}=C_{\mu i}^{\prime} C_{\nu i}^{\prime}, \\
\Theta_{\alpha \beta}^{(i j)}=\sum_{a b} C_{\alpha a}^{\prime} C_{\beta b}^{\prime} \Theta_{i j}^{a b} .
\end{gathered}
$$

We note that the evaluation of $L_{\Theta}^{(x)}$ could also be carried out based on the virtual partitioning scheme, but in practice the occupied partitioning scheme used above is more convenient since the number of matrices $\mathbf{C}^{(i j)}$ and $\boldsymbol{\Theta}^{(i j)}$ for fragment $\mathbf{P}$ (pair fragment PQ) is only the number of occupied orbitals assigned to atomic site $\mathrm{P}$ (atomic sites $\mathrm{P}$ or $\mathrm{Q}$ ) squared. If instead the virtual partitioning scheme were invoked for $L_{\Theta}^{(x)}$ the corresponding number of matrices $\mathbf{C}^{(a b)}$ and $\boldsymbol{\Theta}^{(a b)}$ would be the significantly larger number of virtual orbitals assigned to atomic site $\mathrm{P}$ (atomic site $\mathrm{P}$ or $\mathrm{Q}$ ) squared. We emphasize, however, that the other intermediates $(\mathbf{X}, \mathbf{Y}$, and $\boldsymbol{\Phi})$ are inherently tied to either the occupied or the virtual partitioning scheme.

The precision of the DEC-MP2 molecular gradient compared to a standard MP2 gradient calculation was discussed in Sec. III A (see the discussion following Eq. (75)). It was argued that in the DEC scheme the FOT - which directly determines the precision of the Lagrangian correlation energy by optimizing the size of the AOS in a black-box manner indirectly determines the precision of the MP2 amplitudes, and thus the precision of the MP2 molecular gradient. Having presented the equations that define the DEC-MP2 molecular gradient in this section we can now see this more explicitly. Two main sources of errors for the fragment energies were listed in Sec. III A: (i) the summations in the fragment energy expressions run only over orbitals inside the AOS, (ii) the MP2 amplitude equation is solved in the AOS rather than the full orbital space. From the equations in this section it is clear that errors of type (i) and (ii) also occur for the gradient intermediate fragment contributions $\mathbf{X}_{\mathrm{P}}, \mathbf{Y}_{\mathrm{P}}, \boldsymbol{\Phi}_{\mathrm{P}}, L_{\Theta, \mathrm{P}}^{(x)}$, $\Delta \mathbf{X}_{\mathrm{PQ}}, \Delta \mathbf{Y}_{\mathrm{PQ}}, \Delta \boldsymbol{\Phi}_{\mathrm{PQ}}$, and $\Delta L_{\Theta, \mathrm{PQ}}^{(x)}$ (compare, for example, the full molecular expression in Eq. (77) to the DEC expression in Eq. (79)). The FOT therefore indirectly determines the precision of the gradient intermediates. For example, if the FOT is lowered, the AOS will increase to include additional amplitudes and integrals to adjust to the requested precision. In this way errors (i) and (ii) will both decrease, and the gradient intermediates (and therefore the molecular gradient itself) will be determined with higher precision. In summary, the single input threshold FOT explicitly determines the precision of the correlation energy by optimizing the fragment orbital spaces in a black-box manner according to an energy criterion, and thereby the FOT implicitly determines the precision of the MP2 molecular gradient.

\section{Error estimate using the MP2 correlation density matrix}

In this section we describe how the trace of the MP2 correlation density matrix $\rho$ in Eq. (38) provides us with a simple measure of the fragmentation errors in a DEC calculation. One might argue that this measure is superfluous since, by construction, the FOT defines the precision of a DEC calculation. However, $\operatorname{Tr} \rho$ may serve as a consistency check to validate the result of a DEC calculation, also in cases where a full molecular reference calculation cannot be carried out.

In a full calculation the trace of the correlation density matrix $\rho$ in Eq. (38) is zero as may easily be verified using Eqs. (35) and (36),

$$
\begin{aligned}
\operatorname{Tr} \rho & =\sum_{q} \rho_{q q}=\sum_{a} Y_{a a}-\sum_{i} X_{i i} \\
& =\sum_{a} \sum_{b i j} t_{j i}^{b a} \bar{t}_{j i}^{b a}-\sum_{i} \sum_{a b j} t_{j i}^{b a} \bar{t}_{j i}^{b a}=0 .
\end{aligned}
$$

Equation (115) simply states that the MP2 correlation treatment conserves the number of electrons. No assumptions about the amplitudes were used to obtain Eq. (115), and therefore $\operatorname{Tr} \rho$ is exactly zero in approaches where a single set of amplitudes is used. For example, $\operatorname{Tr} \rho$ is trivially zero in local MP2 correlation methods using a single set of approximate amplitudes, regardless of the resemblance of the approximate MP2 amplitudes with the exact MP2 amplitudes. Tr $\rho$ is therefore not a useful quantity in local MP2 correlation methods where a single set of approximate amplitudes is employed. In 
contrast, in the DEC scheme the occupied and virtual partitioning schemes define two different sets of amplitudes, the "occupied partitioning amplitudes" (OPAs) and the "virtual partitioning amplitudes" (VPAs). In the limit of a full calculation these two sets are of course identical but when DEC locality approximations are introduced the OPAs and VPAs will in general be different. When $\operatorname{Tr} \rho$ is evaluated using the DEC scheme, the OPAs are used to evaluate the $Y$ contributions, while the VPAs are used for the $X$ contributions as described in Sec. III C. $\operatorname{Tr} \rho$ is therefore a simple measure of how much the OPAs differ from the VPAs, which, in turn, may be used as an internal consistency check of the precision of the calculation. To see this explicitly we use Eqs. (79), (80), (84), and (85) to obtain the following expression for $\operatorname{Tr} \rho$ in a DEC calculation:

$$
\begin{aligned}
\operatorname{Tr} \rho= & \sum_{a} Y_{a a}-\sum_{i} X_{i i} \\
= & \left.\sum_{a}\left(\sum_{\mathrm{P}}\left(Y_{\mathrm{P}}\right)_{a a}+\sum_{P>Q} \Delta Y_{\mathrm{PQ}}\right)_{a a}\right) \\
& \left.-\sum_{i}\left(\sum_{\mathrm{P}}\left(X_{\mathrm{P}}\right)_{i i}+\sum_{P>Q} \Delta X_{\mathrm{PQ}}\right)_{i i}\right) \\
= & \sum_{\mathrm{P}} \sum_{i j \in \mathrm{P}} \sum_{a b \in[\overline{\mathrm{P}}]}\left(t_{\mathrm{P}}\right)_{j i}^{b a}\left(\bar{t}_{\mathrm{P}}\right)_{j i}^{b a} \\
& +\left(\sum_{\substack{i \in \mathrm{P} \\
j \in \mathrm{Q}}}+\sum_{\substack{i \in \mathrm{Q} \\
j \in \mathrm{P}}} \sum_{a b \in[\overline{\mathrm{P}}] \cup[\overline{\mathrm{Q}}]}\left(t_{\mathrm{PQ}}\right)_{j i}^{b a}\left(\bar{t}_{\mathrm{PQ}}\right)_{j i}^{b a}\right. \\
& -\sum_{\mathrm{P}} \sum_{a b \in \mathrm{P}} \sum_{i j \in[\underline{\mathrm{P}}]}\left(t_{\mathrm{P}}\right)_{j i}^{b a}\left(\bar{t}_{\mathrm{P}}\right)_{j i}^{b a} \\
& +\left(\sum_{\substack{a \in \mathrm{P} \\
b \in \mathrm{Q}}}-\sum_{\substack{a \in \mathrm{Q} \\
b \in \mathrm{P}}} \sum_{i j \in[\underline{\mathrm{P}}] \cup[\underline{\mathrm{Q}}]}\left(t_{\mathrm{PQ}}\right)_{j i}^{b a}\left(\bar{t}_{\mathrm{PQ}}\right)_{j i}^{b a},\right.
\end{aligned}
$$

where we have introduced an amplitude/multiplier subscript $\mathrm{P}$ (PQ) to emphasize that the amplitudes/multipliers are obtained in a specific atomic fragment $\mathrm{P}$ (pair fragment PQ) calculation. The first two terms involve OPAs, while the two last terms involve VPAs.

Clearly, Eq. (116) reduces to Eq. (115) in the limit where the fragment spaces $[\mathrm{P}]$ and $[\overline{\mathrm{P}}]$ include the full orbital space for all atoms $\mathrm{P}$, and all amplitudes are determined in the same full molecular calculation. However, in a DEC calculation, the $Y$ contributions in Eq. (116) do not cancel the $X$ contributions exactly. As an example, consider the case where the occupied orbitals $\phi_{k}$ and $\phi_{l}$ are assigned to atomic site $\mathrm{R}$, and the virtual orbitals $\phi_{c}$ and $\phi_{d}$ are assigned to an atomic site $\mathrm{S} \neq \mathrm{R}$. In this case the locality restrictions in Eqs. (65) and (67) imply that

$$
\begin{aligned}
& k l \in \mathrm{R}:\left(t_{\mathrm{R}}\right)_{l k}^{a b} \neq 0 \Rightarrow a b \in[\overline{\mathrm{R}}] \\
& c d \in \mathrm{S}:\left(t_{\mathrm{S}}\right)_{i j}^{d c} \neq 0 \Rightarrow i j \in[\underline{\mathrm{S}}]
\end{aligned}
$$

for general orbitals $\phi_{a}, \phi_{b}, \phi_{i}$, and $\phi_{j}$.
The specific contribution to Eq. (116) from the klcd combination of orbitals will be denoted $A_{d c}^{l k}$, i.e.,

$$
A_{d c}^{l k}=\left(t_{\mathrm{R}}\right)_{l k}^{d c}\left(\bar{t}_{\mathrm{R}}\right)_{l k}^{d c}-\left(t_{\mathrm{S}}\right)_{l k}^{d c}\left(\bar{t}_{\mathrm{S}}\right)_{l k}^{d c}, \quad(k l \in \mathrm{R}, c d \in \mathrm{S}) .
$$

In a full molecular calculation $t_{\mathrm{R}}=t_{\mathrm{S}}$ and $A_{d c}^{l k}=0$. However, due to the locality restrictions in Eqs. (117) and (118), $A_{d c}^{l k}$ is, in general, non-zero. In particular, depending on whether the orbitals $c d(k l)$ are included in $[\overline{\mathrm{R}}]([\underline{\mathrm{S}}])$ or not, there are four different cases:

(a) $\quad c d \in[\overline{\mathrm{R}}], k l \notin[\underline{\mathrm{S}}]: A_{d c}^{l k}=\left(t_{\mathrm{R}}\right)_{l k}^{d c}\left(\bar{t}_{\mathrm{R}}\right)_{l k}^{d c}$

(b) $\quad c d \notin[\overline{\mathrm{R}}], k l \in[\underline{\mathrm{S}}]: A_{d c}^{l k}=-\left(t_{\mathrm{S}}\right)_{l k}^{d c}\left(\bar{t}_{\mathrm{S}}\right)_{l k}^{d c}$

(c) $\quad c d \notin[\overline{\mathrm{R}}], k l \notin[\underline{\mathrm{S}}]: A_{d c}^{l k}=0$

(d) $\quad c d \in[\overline{\mathrm{R}}], k l \in[\underline{\mathrm{S}}]: A_{d c}^{l k}=\left(t_{\mathrm{R}}\right)_{l k}^{d c}\left(\bar{t}_{\mathrm{R}}\right)_{l k}^{d c}-\left(t_{\mathrm{S}}\right)_{l k}^{d c}\left(\overline{\mathrm{t}}_{\mathrm{S}}\right)_{l k}^{d c}$.

In cases (a) and (b) the klcd contributions do not cancel because the summations in the $Y$ - and $X$-contributions of Eq. (116) run differently due to the different sizes of the orbital spaces. In case (d) the terms will, in general, not cancel either because the amplitudes (and multipliers) $t_{\mathrm{R}}$ and $t_{\mathrm{S}}$ have been determined in different fragment orbital spaces since, in general, $[\underline{R}] \neq[\underline{\mathrm{S}}]$ and $[\overline{\mathrm{R}}] \neq[\overline{\mathrm{S}}]$. The deviation of $\operatorname{Tr} \rho$ from zero is therefore a simple measure of both types of errors (i) and (ii) discussed in Sec. III A, i.e., case (a) and (b) are of error type (i), and case (d) is of error type (ii). In conclusion, $\operatorname{Tr} \rho$ probes the different errors in a DEC calculation and is therefore expected to be roughly proportional to the FOT, which defines the precision of the calculation.

\section{ILLUSTRATIVE RESULTS}

It has previously been shown that the DEC-MP2 correlation energy systematically approaches the standard MP2 correlation energy when increasing the precision of the DEC calculation by lowering the FOT. ${ }^{27-29}$ In this section we present numerical results demonstrating that the error in the DEC molecular gradient compared to the standard MP2 gradient also decreases systematically when the FOT is lowered.

We also compare the DEC-MP2 electric dipole moment to the standard MP2 electric dipole moment. The DEC-MP2 electric dipole moment may be calculated using the same scheme as for the molecular gradient. However, the evaluation of the electric dipole moment does not require the use of perturbation-dependent basis sets, and therefore the terms in Eq. (43) involving the differentiated overlap matrix or differentiated two-electron integrals do not contribute to the electric dipole moment. The electric dipole moment can thus be identified from Eq. (43) by omitting all terms except $L_{1 \text {-el }}^{(x)}$ and $h_{\text {nuc }}^{(x)}$, and then replacing the one-electron integrals in Eq. (44) by electric dipole integrals and replacing $h_{\text {nuc }}^{(x)}$ by the nuclearnuclear contribution to the electric dipole moment.

We let $\Delta_{i}$ denote the error of the $i$ th DEC-MP2 gradient component compared to the standard MP2 gradient:

$$
\Delta_{i}=x_{i}^{\mathrm{dec}}-x_{i}^{\mathrm{standard}},
$$

where $x_{i}^{\mathrm{dec}}$ and $x_{i}^{\text {standard }}$ are the $i$ th components of the DEC gradient vector and the standard MP2 gradient vector, respectively. To quantify the errors we consider the mean error $\bar{\Delta}$ 


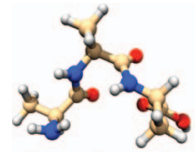

Alanine(3)

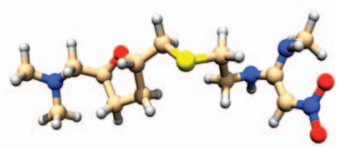

Zantac

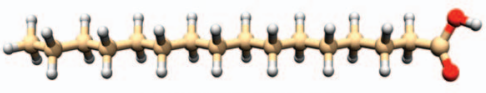

Palmitic acid

FIG. 3. Molecules used in this study.

and the standard deviation $\sigma$,

$$
\begin{gathered}
\bar{\Delta}=\frac{1}{3 N} \sum_{i=1}^{3 N} \Delta_{i}, \\
\sigma=\sqrt{\frac{\sum_{i=1}^{3 N}\left(\Delta_{i}-\bar{\Delta}\right)^{2}}{3 N-1},}
\end{gathered}
$$

where the summations run over all gradient components $(N$ is the number of atoms). Similar error measures are used for the DEC-MP2 dipole moment, where the summations run over the $x, y$, and $z$ components of the dipole moment, rather than over the $3 N$ nuclear coordinates.

Our test molecules are shown in Figure 3, and we consider calculations using the cc-pVDZ and cc-pVTZ basis sets $^{79}$ for FOTs from $10^{-3}$ to $10^{-6}$ a.u. The local orbitals were obtained by minimizing powers of the orbital variance as described in Ref. 30 (in practice a power $m=2$ was used). The calculations have been carried out using a local version of the LSDALTON program. ${ }^{80}$

\section{A. DEC errors as function of the FOT}

Tables I and II contain errors of DEC-MP2 calculations compared to standard MP2 calculations for different FOTs for zantac and alanine(3), respectively. We present the error of the correlation energy $\delta E$, the error measure $\operatorname{Tr} \rho$ discussed in Sec. III D divided by the number of electrons $N_{\mathrm{el}}$ in the system (to obtain a size-intensive error measure), and the standard deviations for the molecular gradient $\sigma$ (grad) and electric dipole moment $\sigma$ (dip). For the electric dipole moment we have also listed the mean error $\bar{\Delta}$ (dip). The mean error for the molecular gradient $\bar{\Delta}$ (grad) is not shown because it is zero by construction (the sum of all gradient components is always zero). In general, the errors in Tables I and II decrease as the FOT is lowered confirming that the FOT indirectly determines the precision of the molecular gradient and electric dipole moment. While $\delta E, \sigma(\mathrm{grad})$, and $\sigma(\mathrm{dip})$ are always positive, $\operatorname{Tr} \rho / N_{\mathrm{el}}$ and $\bar{\Delta}(\mathrm{dip})$ may change sign as the FOT is changed. The convergence of $\operatorname{Tr} \rho / N_{\mathrm{el}}$ and $\bar{\Delta}(\mathrm{dip})$ as the FOT is decreased is therefore less homogeneous than is the case for $\delta E$, $\sigma(\mathrm{grad})$, and $\sigma(\mathrm{dip})$. Nonetheless, it is seen that $\operatorname{Tr} \rho / N_{\mathrm{el}}$ is a useful size-intensive measure of the precision of the calculation, and it may thus be used as a consistency check to validate the precision of DEC calculations on large molecular systems where a standard MP2 reference calculation cannot be carried out.
TABLE I. Errors in DEC-MP2 calculations compared to standard MP2 calculations for different fragment optimization thresholds (FOTs). The calculations were carried out on the zantac molecule using the cc-pVDZ and cc-pVTZ basis sets.

(a) cc-pVDZ basis

\begin{tabular}{llllll}
\hline FOT & \multicolumn{1}{c}{$\delta E^{\mathrm{a}}$} & \multicolumn{1}{c}{$\operatorname{Tr} \rho / N_{\mathrm{el}}^{\mathrm{b}}$} & $\sigma(\mathrm{grad})^{\mathrm{c}}$ & $\sigma(\mathrm{dip})^{\mathrm{d}}$ & $\bar{\Delta}(\mathrm{dip})^{\mathrm{e}}$ \\
\hline $10^{-3}$ & 16 & $-4.2 \times 10^{-5}$ & 0.69 & 26 & 7.7 \\
$10^{-4}$ & 0.95 & $-3.4 \times 10^{-6}$ & 0.079 & 2.9 & -0.72 \\
$10^{-5}$ & 0.065 & $-2.6 \times 10^{-7}$ & 0.0095 & 0.11 & -0.036 \\
$10^{-6}$ & 0.0065 & $-1.7 \times 10^{-8}$ & 0.0012 & 0.020 & -0.0076
\end{tabular}

(b) cc-pVTZ basis

\begin{tabular}{llllll}
\hline FOT & $\delta E$ & $\operatorname{Tr} \rho / N_{\mathrm{el}}$ & $\sigma(\mathrm{grad})$ & $\sigma(\mathrm{dip})$ & $\bar{\Delta}(\mathrm{dip})$ \\
\hline $10^{-3}$ & 9.8 & $-4.0 \times 10^{-5}$ & 1.5 & 6.3 & 1.1 \\
$10^{-4}$ & 0.91 & $-4.5 \times 10^{-6}$ & 0.45 & 1.6 & 0.54 \\
$10^{-5}$ & 0.069 & $-2.7 \times 10^{-7}$ & 0.027 & 0.071 & 0.0054 \\
$10^{-6}$ & 0.0075 & $-2.3 \times 10^{-8}$ & 0.0083 & 0.012 & 0.0020 \\
\hline \hline
\end{tabular}

${ }^{a}$ DEC-MP2 correlation energy error $\left(10^{-3}\right.$ a.u. $)$.

${ }^{\mathrm{b}}$ Error measure discussed in Sec. III D divided by the number of electrons $\left(N_{\mathrm{el}}=172\right)$. ${ }^{\mathrm{c}}$ Standard deviation for DEC-MP2 molecular gradient (mHartree/bohr).

${ }^{\mathrm{d}}$ Standard deviation for DEC-MP2 electric dipole moment $\left(10^{-3}\right.$ a.u. $)$.

${ }^{\mathrm{e}}$ Mean error for DEC-MP2 electric dipole moment ( $10^{-3}$ a.u.).

\section{B. Effect of neglecting distant pairs}

Pair interaction energies $\Delta L_{\mathrm{PQ}}$ describe dispersion effects which decay rapidly with pair distance $R_{\mathrm{PQ}}$ as $R_{\mathrm{PQ}}^{-6}$. Distant pairs may therefore be neglected in DEC-MP2 energy calculations without affecting the precision of the correlation energy as shown previously. ${ }^{28,29}$ The $R_{\mathrm{PQ}}^{-6}$ decay of pair interaction energies is ultimately caused by the decays of the integrals $g_{a i b j}$ and amplitudes $t_{i j}^{a b}$ with distance between the local $\phi_{i} \phi_{a}$ and $\phi_{j} \phi_{b}$ overlap distributions. ${ }^{29}$ Since local amplitudes, local integrals, and local integral derivatives are used to evaluate the DEC-MP2 molecular gradient, distant pairs can also be neglected in DEC-MP2 molecular gradient

TABLE II. Errors in DEC-MP2 calculations compared to standard MP2 calculations for different fragment optimization thresholds (FOTs). The calculations were carried out on the alanine(3) molecule using the cc-pVDZ and cc-pVTZ basis sets.

(a) cc-pVDZ basis

\begin{tabular}{cccccc}
\hline FOT & \multicolumn{1}{c}{$\delta E^{\mathrm{a}}$} & \multicolumn{1}{c}{$\operatorname{Tr} \rho / N_{\mathrm{el}}^{\mathrm{b}}$} & $\sigma(\operatorname{grad})^{\mathrm{c}}$ & $\sigma(\operatorname{dip})^{\mathrm{d}}$ & $\bar{\Delta}(\mathrm{dip})^{\mathrm{e}}$ \\
\hline $10^{-3}$ & 11 & $7.7 \times 10^{-6}$ & 0.78 & 14 & 8.4 \\
$10^{-4}$ & 1.2 & $-2.4 \times 10^{-6}$ & 0.090 & 1.5 & 0.37 \\
$10^{-5}$ & 0.099 & $-3.4 \times 10^{-7}$ & 0.018 & 0.41 & -0.12 \\
$10^{-6}$ & 0.0061 & $-1.6 \times 10^{-8}$ & 0.0019 & 0.043 & 0.0070
\end{tabular}

(b) cc-pVTZ basis

\begin{tabular}{ccclcc}
\hline FOT & $\delta E$ & \multicolumn{1}{c}{$\operatorname{Tr} \rho / N_{\mathrm{el}}$} & $\sigma(\mathrm{grad})$ & $\sigma(\mathrm{dip})$ & $\bar{\Delta}(\mathrm{dip})$ \\
\hline $10^{-3}$ & 13 & $-4.7 \times 10^{-5}$ & 1.4 & 22 & 1.8 \\
$10^{-4}$ & 0.96 & $-5.5 \times 10^{-6}$ & 0.49 & 1.9 & -1.0 \\
$10^{-5}$ & 0.10 & $-4.0 \times 10^{-7}$ & 0.038 & 0.19 & -0.045 \\
$10^{-6}$ & 0.0072 & $-6.2 \times 10^{-8}$ & 0.0019 & 0.019 & 0.035 \\
\hline
\end{tabular}

${ }^{\mathrm{a} D E C}-\mathrm{MP} 2$ correlation energy error $\left(10^{-3}\right.$ a.u.).

${ }^{\mathrm{b}}$ Error measure discussed in Sec. III D divided by the number of electrons $\left(N_{\mathrm{el}}=124\right)$.

${ }^{\mathrm{c}}$ Standard deviation for DEC-MP2 molecular gradient (mHartree/bohr).

${ }^{\mathrm{d}}$ Standard deviation for DEC-MP2 electric dipole moment $\left(10^{-3}\right.$ a.u.).

${ }^{\mathrm{e}}$ Mean error for DEC-MP2 electric dipole moment $\left(10^{-3}\right.$ a.u. $)$. 
TABLE III. DEC-MP2 correlation energy error $\delta E\left(10^{-3}\right.$ a.u. $)$ compared to standard MP2 calculation for different pair cut-off distances $R_{\mathrm{thr}}$ and different fragment optimization thresholds (FOTs) for palmitic acid (cc-pVDZ basis). The number of pair fragments is also shown.

\begin{tabular}{lccccc}
\hline \hline $\begin{array}{l}R_{\text {thr }} \\
(\AA)\end{array}$ & $\begin{array}{c}\text { FOT }=10^{-3} \\
\text { a.u. }\end{array}$ & $\begin{array}{c}\text { FOT }=10^{-4} \\
\text { a.u. }\end{array}$ & $\begin{array}{c}\text { FOT }=10^{-5} \\
\text { a.u. }\end{array}$ & $\begin{array}{c}\text { FOT }=10^{-6} \\
\text { a.u. }\end{array}$ & $\begin{array}{c}\text { No. of } \\
\text { pairs }\end{array}$ \\
\hline 0 & 646 & 632 & 631 & 631 & 0 \\
2 & 120 & 101 & 100 & 100 & 17 \\
4 & 24 & 3.7 & 2.9 & 2.8 & 49 \\
6 & 22 & 1.6 & 0.79 & 0.65 & 65 \\
8 & 22 & 1.1 & 0.22 & 0.083 & 88 \\
10 & 22 & 1.1 & 0.17 & 0.036 & 101 \\
12 & 22 & 1.0 & 0.15 & 0.016 & 119 \\
14 & 22 & 1.0 & 0.15 & 0.012 & 133 \\
16 & 22 & 1.0 & 0.15 & 0.011 & 141 \\
All pairs & 22 & 1.0 & 0.15 & 0.011 & 153 \\
\hline \hline
\end{tabular}

calculations without affecting the precision. To illustrate this matter we now consider DEC-MP2 calculations on the palmitic acid molecule in Figure 3 using different pair cut-off distances $R_{\mathrm{thr}}$ - i.e., for a given $R_{\mathrm{thr}}$ value all atomic pairs $\mathrm{PQ}$ with $R_{\mathrm{PQ}}>R_{\text {thr }}$ have simply been omitted from the DEC calculation. Since there are 18 non-hydrogen atoms in palmitic acid, there are 18 atomic fragments and $18 \times 17 / 2=153$ pair fragments if all pairs are included $\left(R_{\mathrm{thr}}=\infty\right)$. We note that the maximum gradient component in the standard MP2 calculation on palmitic acid is $17.1 \mathrm{mHartree} / \mathrm{bohr}$, and that 94 of the 150 gradient components are larger than one tenth of this maximum value.

In Table III the energy errors $\delta E$ for different FOT and $R_{\text {thr }}$ values are given, and in Table IV the standard deviation $\sigma(\mathrm{grad})$ and maximum error for the individual gradient components $\max \left(\left|\Delta_{i}\right|\right)$ are presented. Considering first the rows with all pairs included it is seen that the error measures decay with decreasing FOT in the same way as in Tables I and II. The rows with all pairs included thus describe the intrinsic error associated with a given FOT. When an $R_{\mathrm{thr}}$ cut-off value is used we in general observe errors which are larger than the intrinsic FOT error. In the extreme case where no pairs are included $\left(R_{\mathrm{thr}}=0\right)$ the errors are roughly independent of the FOT. This just shows that the error associated with a given FOT is much smaller than the huge error introduced when all pairs are omitted. When $R_{\mathrm{thr}}$ is increased for a given FOT, the errors converges to the intrinsic FOT error. Two important conclusions can be drawn by analyzing the convergences of the errors in Tables III and IV.

First, for larger FOT values, the errors converge more rapidly with $R_{\text {thr }}$. For example, for FOT $=10^{-3}$ a.u. and FOT $=10^{-6}$ a.u. the $\max \left(\left|\Delta_{i}\right|\right)$ errors are converged at $R_{\mathrm{thr}}=4 \AA$ and $R_{\mathrm{thr}}=10 \AA$, respectively. This is rather obvious since the error of a DEC calculation increases when the FOT value increases, and calculations using larger FOT values are thus less sensitive to small contributions from widely separated pair fragments.

Second, the gradient error measures (Table IV) converge more rapidly with $R_{\text {thr }}$ than the energy errors (Table III). For example, for FOT $=10^{-5}$ the energy errors and gradient standard deviation are converged at $R_{\mathrm{thr}}=12 \AA$ and $R_{\mathrm{thr}}=6 \AA$,
TABLE IV. DEC-MP2 gradient standard deviation (a) and maximum gradient error (b) compared to standard MP2 calculation for different pair cutoff distances $R_{\text {thr }}$ and different fragment optimization thresholds (FOTs) for palmitic acid (cc-pVDZ basis). The number of pair fragments is also shown.

(a) Gradient standard deviation $\sigma$ (grad) (mHartree/bohr)

\begin{tabular}{lccccr}
\hline $\begin{array}{l}R_{\mathrm{thr}} \\
(\AA)\end{array}$ & $\begin{array}{c}\text { FOT }=10^{-3} \\
\text { a.u. }\end{array}$ & $\begin{array}{c}\text { FOT }=10^{-4} \\
\text { a.u. }\end{array}$ & $\begin{array}{c}\text { FOT }=10^{-5} \\
\text { a.u. }\end{array}$ & $\begin{array}{c}\text { FOT }=10^{-6} \\
\text { a.u. }\end{array}$ & $\begin{array}{r}\text { No. of } \\
\text { pairs }\end{array}$ \\
\hline 0 & 2.7 & 2.8 & 2.8 & 2.8 & 0 \\
2 & 0.98 & 0.92 & 0.94 & 0.94 & 17 \\
4 & 0.43 & 0.11 & 0.031 & 0.022 & 49 \\
6 & 0.43 & 0.12 & 0.027 & 0.0063 & 65 \\
8 & 0.43 & 0.12 & 0.027 & 0.0027 & 88 \\
10 & 0.43 & 0.12 & 0.027 & 0.0026 & 101 \\
12 & 0.43 & 0.12 & 0.027 & 0.0026 & 119 \\
14 & 0.43 & 0.12 & 0.027 & 0.0026 & 133 \\
16 & 0.43 & 0.12 & 0.027 & 0.0026 & 141 \\
All pairs & 0.43 & 0.12 & 0.027 & 0.0026 & 153
\end{tabular}

(b) Maximum gradient error, $\max \left(\left|\Delta_{i}\right|\right)(\mathrm{mHartree} / \mathrm{bohr})$

\begin{tabular}{lccccc}
\hline $\begin{array}{l}R_{\text {thr }} \\
(\AA)\end{array}$ & $\begin{array}{c}\text { FOT }=10^{-3} \\
\text { a.u. }\end{array}$ & $\begin{array}{c}\text { FOT }=10^{-4} \\
\text { a.u. }\end{array}$ & $\begin{array}{c}\text { FOT }=10^{-5} \\
\text { a.u. }\end{array}$ & $\begin{array}{c}\text { FOT }=10^{-6} \\
\text { a.u. }\end{array}$ & $\begin{array}{c}\text { No. of } \\
\text { pairs }\end{array}$ \\
\hline 0 & 15 & 15 & 15 & 15 & 0 \\
2 & 4.1 & 3.9 & 3.6 & 3.7 & 17 \\
4 & 1.6 & 0.52 & 0.11 & 0.10 & 49 \\
6 & 1.6 & 0.50 & 0.12 & 0.029 & 65 \\
8 & 1.6 & 0.49 & 0.12 & 0.016 & 88 \\
10 & 1.6 & 0.48 & 0.12 & 0.014 & 101 \\
12 & 1.6 & 0.48 & 0.12 & 0.014 & 119 \\
14 & 1.6 & 0.48 & 0.12 & 0.014 & 133 \\
16 & 1.6 & 0.48 & 0.12 & 0.014 & 141 \\
All pairs & 1.6 & 0.48 & 0.12 & 0.014 & 153 \\
\hline \hline
\end{tabular}

respectively. To understand this behaviour recall that the pair interaction energies $\Delta L_{\mathrm{PQ}}$ decay with the inverse pair distance to the sixth power, i.e., $\Delta L_{\mathrm{PQ}} \propto R_{\mathrm{PQ}}^{-6}$. The gradient of a dispersion energy contribution $d \Delta L_{\mathrm{PQ}} / d R_{\mathrm{PQ}}$ will therefore decay more rapidly as $R_{\mathrm{PQ}}^{-7}$, and distant pairs thus have a smaller effect on the molecular gradient than on the energy. In a practical calculation where only the gradient is of interest (for example, in the context of a geometry optimization), a smaller $R_{\text {thr }}$ value can be used than would be the case in a calculation where the correlation energy is targeted.

Optimally, the error introduced by applying a $R_{\mathrm{thr}}$ cut-off should be insignificant compared to the intrinsic FOT error - but at the same time $R_{\mathrm{thr}}$ should be as small as possible to minimize the computational effort. The decays of the errors in Tables III and IV with $R_{\text {thr }}$ indicate that it is possible to determine the optimal $R_{\text {thr }}$ value in a systematic way during the calculation. For example, for a given molecule and a given FOT, one may compare the calculated correlation energies (and/or molecular gradients) for different $R_{\mathrm{thr}}$ values to ensure that the calculation is converged with respect to the inclusion of pair fragments. Such a scheme can be applied in a black box manner to a general molecule due to the universal $R_{\mathrm{PQ}}^{-6}$ behaviour of dispersion energies and $R_{\mathrm{PQ}}^{-7}$ decay for gradient dispersion contributions. In this manner $R_{\mathrm{thr}}$ will be determined in a self-adaptive manner during the calculation rather than being an input threshold. In general, the optimal 
$R_{\text {thr }}$ value will increase if a greater precision (smaller FOT) is requested. A detailed procedure for the self-adaptive determination of the optimal $R_{\mathrm{thr}}$ value will be presented in a forthcoming paper.

\section{Cost and memory analysis}

In this section we comment on the cost and memory requirements of a DEC-MP2 calculation compared to a standard MP2 calculation. As test systems we consider a series of fatty acids $\mathrm{C}_{n} \mathrm{O}_{2} \mathrm{H}_{2 n}$ for $n=16,32,48,64,128$. The fatty acid with $n=16$ is palmitic acid in Figure 3 and the remaining fatty acids are simple extensions of this structure. We use FOT $=10^{-4}$ a.u., $R_{\mathrm{thr}}=10 \AA$, and the cc-pVDZ basis set in the examples considered.

A standard MP2 implementation formally scales as $\mathcal{O}\left(N^{5}\right)$ where $N$ is a measure of the system size. In particular, for large systems, the most expensive step is to transform the first $\mathrm{AO}$ index in the two-electron integrals to an occupied MO index. This procedure scales as $\mathcal{O}\left(O M^{4}\right)$ where $O$ and $M$ denote the number of occupied orbitals and the number of atomic basis functions, respectively. In Figure $4, O M^{4}$ operation counts are given for standard and DEC calculations relative to the standard calculation for $\mathrm{C}_{16} \mathrm{O}_{2} \mathrm{H}_{32}$. We note that each DEC fragment calculation effectively corresponds to a small standard MP2 calculation performed in the AOS using the atomic basis functions in the atomic fragment extent, see Figure 1. The DEC numbers in Figure 4 were therefore determined by adding up $O_{\mathrm{AOS}} M_{\mathrm{AE}}^{4}$ for all fragments, where, for each fragment, $O_{\mathrm{AOS}}$ and $M_{\mathrm{AE}}$ denote the number of occupied AOS orbitals and the number of atomic basis functions in the atomic fragment extent, respectively.

Two competing effects decide whether a standard or a DEC calculation has the highest operation count. DEC is favorable compared to a standard calculation because all fragment calculations use a small subset of the full molecular orbital space. On the other hand, in a DEC calculation there are significant overlaps between the orbital spaces of neighbouring fragments, and the same operations are therefore repeated

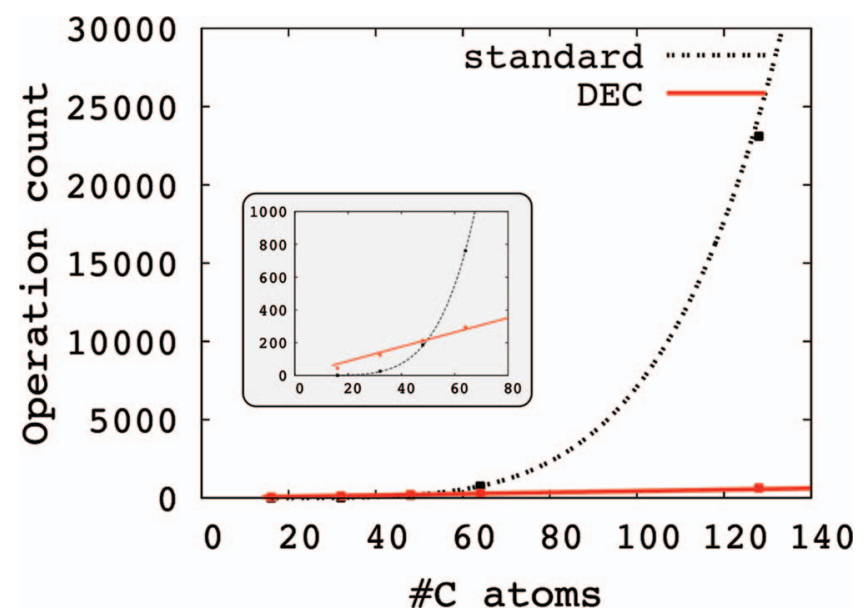

FIG. 4. Operation counts for the dominating MP2 step for standard (black double-dashed) and DEC (red solid) calculations for fatty acids $\mathrm{C}_{n} \mathrm{O}_{2} \mathrm{H}_{2 n}$ ( $n=16,32,48,64,128)$ using the cc-pVDZ basis set. The numbers are given relative to the standard MP2 calculation on $\mathrm{C}_{16} \mathrm{O}_{2} \mathrm{H}_{32}$. many times, which is clearly disadvantageous compared to a standard implementation. For $\mathrm{C}_{16} \mathrm{O}_{2} \mathrm{H}_{32}$ the DEC operation count is significantly higher than the standard operation count (by a factor $\approx 45$ ). Thus, for $\mathrm{C}_{16} \mathrm{O}_{2} \mathrm{H}_{32}$ the repetition overhead clearly outweighs the savings obtained by using small orbital spaces. However, as the molecular size is increased, the DEC operation count increases only linearly as with system size, while the standard operation count increases with the system size to the fifth power. Thus, at some point there is a crossover where the DEC operation count is smaller than the standard operation count. For the systems considered in Figure 4 this happens roughly at $\mathrm{C}_{50} \mathrm{O}_{2} \mathrm{H}_{100}$. In the more extreme case of $\mathrm{C}_{128} \mathrm{O}_{2} \mathrm{H}_{256}$ the standard calculation becomes very expensive, while the DEC calculation is still doable with modest computational resources.

Figure 4 summarizes the total operation count for all fragment calculations, but it tells nothing about parallel efficiency. As discussed previously, the DEC approach is embarrassingly parallel because the fragment calculations are independent. Furthermore, in a forthcoming paper we demonstrate that the individual DEC fragment calculations may also be efficiently parallelized. The parallel scalability of the DEC scheme is thus very favorable and highly suited for large super computer architectures. In practice, the two levels of parallelization imply that, if a sufficient number of computer nodes is available, the correlated part of a DEC-MP2 gradient calculation (yellow/dotted boxes in Figure 2) may be performed in a matter of hours for virtually any system size. Strategies for efficient parallelization of also the HF related parts of the DEC-MP2 gradient calculation (blue/solid boxes in Figure 2) are currently being investigated in our laboratory.

Let us finally compare memory (or disk space) requirements in standard MP2 and DEC-MP2 calculations. In a standard MP2 calculation the biggest challenge is to handle integrals of size $O V^{3}$, where $V$ is the number of virtual orbitals, see Eq. (40). In contrast, in a DEC calculation for atomic fragment $\mathrm{P}$ only arrays of size $O_{\mathrm{AOS}} V_{\mathrm{AOS}} V_{\mathrm{P}}^{2}$ is required, where $V_{\mathrm{AOS}}$ is the number of virtual AOS orbitals for fragment $\mathrm{P}$, and $V_{\mathrm{P}}$ is the number of virtual orbitals assigned to atomic site P, see Eqs. (104)-(106) and the accompanying discussion. In general, $V_{\mathrm{P}}$ is of course much smaller than $V_{\mathrm{AOS}}$, see Figure 1. In DEC calculations no four-indexed arrays involve full molecular dimensions, and therefore the DEC memory requirements are much smaller than in standard implementations. Thus, the use of local disks is avoided in the DEC scheme.

TABLE V. Memory (or disk space) required to stored $O V^{3}$ arrays in standard and DEC calculations for fatty acids $\mathrm{C}_{n} \mathrm{O}_{2} \mathrm{H}_{2 n}(n=16,32,48,64,128)$ using the cc-pVDZ basis set. For DEC the required memory for the largest fragment is given.

\begin{tabular}{lcc}
\hline \hline No. of C atoms & Standard memory (GB) & DEC memory (GB) \\
\hline 16 & 22.6 & 0.105 \\
32 & 313 & 0.112 \\
48 & 1510 & 0.112 \\
64 & 4640 & 0.112 \\
128 & 71400 & 0.125 \\
\hline \hline
\end{tabular}


In Table $\mathrm{V}$ we consider the fatty acid series above and present the memory (or disk space) required to store $O V^{3}$ arrays in standard calculations and the maximum memory required in any of the DEC fragment calculations. The standard requirements quickly become prohibitively large and various techniques, e.g., repeated calculation of AO integrals, are required to circumvent this bottleneck. On the contrary, in DEC calculations the maximum memory required during the fragment calculations stays basically constant at $\approx 0.1$ gigabytes (GB) which is easily manageable. The DEC memory requirement is independent of system size because the size of the AOS is system-independent. This is the case because the underlying localized molecular orbitals ${ }^{30}$ have the same locality for all fatty acids. In particular, for all fatty acids considered in this section, the maximum orbital spread was 1.6 a.u. for the occupied orbitals and 2.4 a.u. for the virtual orbitals.

\section{SUMMARY AND FUTURE PERSPECTIVES}

We have demonstrated that the MP2 molecular gradient can be evaluated using the DEC scheme as summarized in Figure 2. All manipulations of four-index quantities are carried out using small local orbital fragment spaces (yellow/dotted boxes in Figure 2), while the HF-like twoindex manipulations involving effective MP2 densities are carried out for the full molecular system (blue/solid boxes in Figure 2). In other words, the short-range electron correlation effects (four-index quantities) are treated locally using orbital fragments, whereas a proper treatment of the long-range electronic interactions (two-index quantities) requires that the full molecular system is considered.

The orbital fragment calculations start out with a set of atomic fragment calculations followed by a set of pair fragment calculations. The total number of atomic and pair fragments to consider scales linearly with system size. Thus, if linear-scaling techniques ${ }^{67-73}$ are employed for the HF-like contributions, the evaluation of the DEC-MP2 molecular gradient as a whole is linear-scaling. The orbital fragment calculations are furthermore embarrassingly parallelizable.

The single threshold FOT controls the precision of the DEC-MP2 Lagrangian energy compared to the full molecular MP2 Lagrangian energy by optimizing the sizes of the orbital fragments in a black box manner. By construction the precision of the DEC-MP2 Lagrangian energy defines the precision of the MP2 amplitudes, which ultimately defines the precision of the DEC-MP2 molecular gradient. Thus, the precision of the DEC-MP2 molecular gradient is implicitly controlled by the FOT, which has also been confirmed by numerical test calculations.

Pair interaction energies $\Delta L_{\mathrm{PQ}}$ have an $R_{\mathrm{PQ}}^{-6}$ distance decay characteristic of dispersion energy contributions. Consequently, pair fragment contributions beyond a given pair distance threshold can be neglected without affecting the precision of the calculated correlation energy, and this is the reason why only a linear-scaling number of pair fragment calculations need to be carried out. The pair interaction energy contributions to the gradient $\left(d \Delta L_{\mathrm{PQ}} / d R_{\mathrm{PQ}}\right)$ have an $R_{\mathrm{PQ}}^{-7}$ distance decay. For molecular gradients, pair fragments may therefore be neglected for pair distance thresholds which are significantly smaller than for the energy. This simplifies the evaluation of the molecular gradient compared to the energy.

An obvious next step is to apply the DEC-MP2 molecular gradient in the context of performing geometry optimizations for large molecular systems. This is a subject for future investigations and requires efficient massive parallelization of all steps in Figure 2 (in our current implementation the blue/solid boxes in Figure 2 are not massively parallel). At this stage we note that it will be advantageous to tighten the FOT during the geometry optimization, such that a more accurate MP2 gradient is obtained when the molecular geometry is close to the equilibrium structure.

The development of the DEC-MP2 molecular gradient is the initial step towards calculating higher order energy derivatives within the DEC framework, both at the MP2 level of theory and for more accurate coupled-cluster methods, such as the CCSD model. The final expression for the MP2 molecular gradient is invariant to the choice of connection matrix $\mathbf{T}(\mathbf{x})$ in Eq. (16). In general, first-order CC properties represented as a Lagrangian derivative $d L /\left.d \boldsymbol{\varepsilon}\right|_{\boldsymbol{\varepsilon}=\boldsymbol{\varepsilon}_{0}}$ (where $\boldsymbol{\varepsilon}$ represents a general perturbation) are invariant to the choice of connection matrix $\mathbf{T}(\boldsymbol{\varepsilon})$, since orbital relaxation parameters $\boldsymbol{\kappa}(\boldsymbol{\varepsilon})$ only need to be determined for the reference system $\left(\varepsilon=\boldsymbol{\varepsilon}_{0}\right)$. However, for second- (and higher-)order molecular properties perturbed orbital relaxation parameters are also required. In a local DEC-CC context it then becomes important to use the natural connection matrix, ${ }^{76}$ which ensures that the OMOs $\hat{\phi}(\boldsymbol{\varepsilon})$ resembles the optimized local HF orbitals $\phi\left(\boldsymbol{\varepsilon}_{0}\right)$ for the reference system as closely as possible. In this way the locality of the reference orbitals is preserved when the perturbation is changed infinitesimally from $\boldsymbol{\varepsilon}_{0} \rightarrow \boldsymbol{\varepsilon}_{0}+\delta \boldsymbol{\varepsilon}$. Thus, using the natural connection for second- (and higher-)order properties, the first- (and higher-)order CC amplitudes and multipliers may be determined using the same local fragment orbital spaces that are used to determine the zeroth-order CC amplitudes and multipliers.

\section{ACKNOWLEDGMENTS}

The research leading to these results has received funding from the European Research Council under the European Union's Seventh Framework Programme (FP/20072013)/ERC Grant Agreement No. 291371. This work has also been supported by the Lundbeck Foundation and the Danish Center for Scientific Computing (DCSC). T.K. acknowledges support from The Danish Council for Independent Research-Natural Sciences. S.R. acknowledges support from the Norwegian Research council through the CoE Centre for Theoretical and Computational Chemistry (CTCC) Grant No. $179568 /$ V30.

\section{APPENDIX: INTERMEDIATES USED FOR THE MP2 GRADIENT}

In Appendix A 1 we list the intermediates introduced to determine the molecular gradient in the AO basis. Appendix A 2 describes how the Coulomb and exchange contributions used for the $\bar{\kappa}$ equation in Eq. (57) are evaluated in the $\mathrm{AO}$ basis and then transformed back to the $\mathrm{MO}$ basis. 
All quantities are implicitly understood to be evaluated at the reference geometry $\mathbf{x}_{0}$.

\section{Intermediates in the $\mathrm{AO}$ basis}

The MP2 correlation density $\rho$ transforms from the MO to the $\mathrm{AO}$ basis according to

$$
\rho_{\mu \nu}=\sum_{p q} C_{\mu p} C_{v q} \rho_{p q}
$$

and likewise for the $\boldsymbol{\Phi}$ matrix.

The standard HF AO density matrix (for a closed-shell system) is given by

$$
D_{\mu \nu}=\sum_{i} C_{\mu i} C_{\nu i}
$$

where the summation runs over all occupied orbitals. The expression for the MP2 gradient in the AO basis also involves an effective density matrix $\overline{\mathbf{D}}$, where the summation in Eq. (A2) runs over both the occupied and the virtual orbitals,

$$
\bar{D}_{\mu \nu}=\sum_{p} C_{\mu p} C_{\nu p}
$$

The reorthonormalization term of the gradient in the AO basis may be written compactly by introducing the following intermediate matrix $\mathbf{W}$,

$$
\mathbf{W}=2 \mathbf{D F D}+\frac{1}{2} \boldsymbol{\Phi}+\boldsymbol{\rho} \mathbf{F} \overline{\mathbf{D}}+\mathbf{D G}(\rho) \overline{\mathbf{D}}
$$

where all matrices are given in the $\mathrm{AO}$ basis. The $\mathbf{G}(\mathbf{A})$ transformation on a general matrix $\mathbf{A}$ is given as the sum of a Coulomb contribution $\mathbf{J}(\mathbf{A})$ and an exchange contribution $\mathbf{K}(\mathbf{A})$,

$$
\begin{gathered}
G_{\mu \nu}(\mathbf{A})=2 J_{\mu \nu}(\mathbf{A})-K_{\mu \nu}(\mathbf{A}), \\
J_{\mu \nu}(\mathbf{A})=\sum_{\alpha \beta} g_{\mu \nu \alpha \beta} A_{\alpha \beta}, \\
K_{\mu \nu}(\mathbf{A})=\sum_{\alpha \beta} g_{\mu \beta \alpha \nu} A_{\alpha \beta} .
\end{gathered}
$$

Similarly, $\mathbf{J}^{(x)}(\mathbf{A})$ and $\mathbf{K}^{(x)}(\mathbf{A})$ refers to coulomb and exchange transformations with differentiated integrals, i.e.,

$$
\begin{aligned}
J_{\mu \nu}^{(x)}(\mathbf{A}) & =\sum_{\alpha \beta} g_{\mu \nu \alpha \beta}^{(x)} A_{\alpha \beta}, \\
K_{\mu \nu}^{(x)}(\mathbf{A}) & =\sum_{\alpha \beta} g_{\mu \beta \alpha \nu}^{(x)} A_{\alpha \beta} .
\end{aligned}
$$

For the $L_{\Theta}^{(x)}$ term the following intermediate matrices are introduced,

$$
\begin{gathered}
C_{\mu \nu}^{(i j)}=C_{\mu i} C_{\nu i}, \\
\Theta_{\alpha \beta}^{(i j)}=\sum_{a b} C_{\alpha a} C_{\beta b} \Theta_{i j}^{a b},
\end{gathered}
$$

where $C_{\mu \nu}^{(i j)}$ should be thought of as a two-index matrix with AO indices $\mu$ and $\nu$, which refers to a specific pair of occupied orbitals $\phi_{i}$ and $\phi_{j}$ (and similarly for $\Theta_{\alpha \beta}^{(i j)}$ ).

\section{Coulomb and exchange transformations for $\bar{\kappa}$ equation}

In practice the Coulomb and exchange transformations entering the $\overline{\boldsymbol{\kappa}}$ equation in Eq. (57) are evaluated in the $\mathrm{AO}$ basis and then transformed back to the MO basis, where Eq. (57) is solved iteratively. Here we consider these transformations in detail.

The Coulomb and exchange transformations on the $\bar{\kappa}$ matrix in Eq. (57) may be written as

$$
2 \sum_{a i} \bar{\kappa}_{a i}\left(4 g_{a i d l}-g_{a d l i}-g_{a l d i}\right)=2 G_{d l}\left(\bar{\kappa}^{\mathrm{s}}\right),
$$

where $\overline{\boldsymbol{\kappa}}$ is a symmetrized $\overline{\boldsymbol{\kappa}}$ matrix in the AO basis,

$$
\bar{\kappa}_{\mu \nu}^{\mathrm{s}}=\sum_{a i}\left(\bar{\kappa}_{a i} C_{\mu a} C_{\nu i}+\bar{\kappa}_{a i} C_{\nu a} C_{\mu i}\right),
$$

and $G_{d l}\left(\overline{\boldsymbol{\kappa}}^{\mathrm{s}}\right)$ is the Fock transformation in Eq. (A5) with the first/second index transformed to the virtual/occupied MO basis,

$$
G_{d l}\left(\overline{\boldsymbol{\kappa}}^{\mathrm{s}}\right)=\sum_{\alpha \beta} C_{\alpha d} C_{\beta l} G_{\alpha \beta}\left(\overline{\boldsymbol{\kappa}}^{\mathrm{s}}\right) .
$$

The Coulomb and exchange transformations in Eq. (57) involving $\mathbf{X}$ and $\mathbf{Y}$ may be combined and evaluated as

$$
\begin{aligned}
& \sum_{i k} X_{i k}\left(4 g_{d l i k}-2 g_{d k i l}\right) \\
& \quad-\sum_{a c} Y_{a c}\left(4 g_{d l a c}-2 g_{d c a l}\right)=2 G_{d l}(\mathbf{M}),
\end{aligned}
$$

where

$$
M_{\mu \nu}=\sum_{i k} C_{\mu i} C_{\nu k} X_{i k}-\sum_{a c} C_{\mu a} C_{\nu c} Y_{a c} .
$$

${ }^{1}$ P. Pulay, Chem. Phys. Lett. 100, 151 (1983).

${ }^{2}$ S. Saebø and P. Pulay, Annu. Rev. Phys. Chem. 44, 213-236 (1993).

${ }^{3}$ C. Hampel and H.-J. Werner, J. Chem. Phys. 104, 6286-6297 (1996).

${ }^{4}$ M. Schütz, G. Hetzer, and H.-J. Werner, J. Chem. Phys. 111, 5691-5705 (1999)

${ }^{5}$ M. Schütz and H.-J. Werner, J. Chem. Phys. 114, 661-681 (2001).

${ }^{6}$ H.-J. Werner and M. Schütz, J. Chem. Phys. 135, 144116 (2011).

${ }^{7}$ G. E. Scuseria and P. Y. Ayala, J. Chem. Phys. 111, 8330-8343 (1999).

${ }^{8}$ O. Christiansen, P. Manninen, P. Jørgensen, and J. Olsen, J. Chem. Phys. 124, 084103 (2006).

${ }^{9}$ V. Weijo, P. Manninen, P. Jørgensen, O. Christiansen, and J. Olsen, J. Chem. Phys. 127, 074106 (2007).

${ }^{10}$ J. E. Subotnik, A. Sodt, and M. Head-Gordon, J. Chem. Phys. 125, 074116 (2006).

${ }^{11}$ N. Flocke and R. J. Bartlett, J. Chem. Phys. 121, 10935-10944 (2004).

${ }^{12}$ S. Li, J. Ma, and Y. Jiang, J. Comput. Chem. 23, 237 (2002).

${ }^{13}$ W. Li, P. Piecuch, J. R. Gour, and S. Li, J. Chem. Phys. 131, 114109 (2009).

${ }^{14} \mathrm{~W}$. Li and P. Piecuch, J. Phys. Chem. A 114, 8644 (2010).

${ }^{15}$ M. Kobayashi and H. Nakai, J. Chem. Phys. 129, 044103 (2008).

${ }^{16}$ D. G. Fedorov and K. Kitaura, J. Chem. Phys. 123, 134103 (2005).

${ }^{17}$ H. Stoll, Chem. Phys. Lett. 191, 548 (1992).

${ }^{18}$ J. Friedrich, M. Hanrath, and M. Dolg, J. Chem. Phys. 126, 154110 (2007).

${ }^{19}$ P. Y. Ayala and G. E. Scuseria, J. Chem. Phys. 110, 3660-3671 (1999).

${ }^{20}$ D. S. Lambrecht, B. Doser, and C. Ochsenfeld, J. Chem. Phys. 123, 184102 (2005).

${ }^{21}$ B. Doser, D. S. Lambrech, and C. Ochsenfeld, Chem. Phys. Phys. Chem. 10, 3335 (2008).

${ }^{22}$ A. A. Auer and M. Nooijen, J. Chem. Phys. 125, 024104 (2006).

${ }^{23}$ F. Neese, F. Wennmohs, and A. Hansen, J. Chem. Phys. 130, 114108 (2009). 
${ }^{24}$ F. Neese, A. Hansen, and D. G. Liakos, J. Chem. Phys. 131, 064103 (2009).

${ }^{25}$ J. Yang, Y. Kurashige, F. R. Manby, and G. K. L. Chan, J. Chem. Phys. 134, 044123 (2011).

${ }^{26}$ J. Yang, G. K. L. Chan, F. R. Manby, M. Schütz, and H.-J. Werner, J. Chem. Phys. 136, 114105 (2012).

${ }^{27}$ M. Ziółkowski, B. Jansík, T. Kjærgaard, and P. Jørgensen, J. Chem. Phys. 133, 014107 (2010).

${ }^{28}$ K. Kristensen, M. Ziółkowski, B. Jansík, T. Kjærgaard, and P. Jørgensen, J. Chem. Theory Comput. 7, 1677 (2011).

${ }^{29}$ I.-M. Høyvik, K. Kristensen, B. Jansík, and P. Jørgensen, J. Chem. Phys. 136, 014105 (2012).

${ }^{30}$ B. Jansík, S. Høst, K. Kristensen, and P. Jørgensen, J. Chem. Phys. 134, 194104 (2011).

${ }^{31}$ P. Pulay, Mol. Phys. 17, 197 (1969).

${ }^{32}$ J. A. Pople, R. Krishnan, H. B. Schlegel, and J. S. Binkley, Int. J. Quantum Chem., Symp. 13, 225 (1979).

${ }^{33}$ G. Fitzgerald, R. Harrison, W. D. Laidig, and R. J. Bartlett, J. Chem. Phys. 82, 4379 (1985).

${ }^{34}$ J. Gauss and D. Cremer, Chem. Phys. Lett. 153, 303 (1988).

${ }^{35}$ B. R. Brooks, W. D. Laidig, P. Saxe, J. D. Goddard, Y. Yamaguchi, and H. F. Schaefer III, J. Chem. Phys. 72, 4652 (1980).

${ }^{36}$ R. Krishnan, H. Schlegel, and J. A. Pople, J. Chem. Phys. 72, 4654 (1980).

${ }^{37}$ J. Gauss and D. Cremer, Chem. Phys. Lett. 150, 280 (1988).

${ }^{38}$ J. Gauss and D. Cremer, Chem. Phys. Lett. 163, 549 (1989).

${ }^{39}$ A. Scheiner, G. E. Scuseria, J. E. Rice, T. J. Lee, and H. F. Schaefer III, J. Chem. Phys. 87, 5361 (1987).

${ }^{40}$ G. E. Scuseria, J. Chem. Phys. 94, 442 (1991).

${ }^{41}$ T. J. Lee and A. P. Rendell, J. Chem. Phys. 94, 6229 (1991).

${ }^{42}$ J. Gauss and J. F. Stanton, Chem. Phys. Phys. Chem. 2, 2047 (2000).

${ }^{43}$ N. C. Handy, R. D. Amos, J. F. Gaw, J. E. Rice, and E. D. Simandiras, Chem. Phys. Lett. 120, 151 (1985).

${ }^{44}$ E. Rice and R. D. Amos, Chem. Phys. Lett. 122, 585 (1985).

${ }^{45}$ E. D. Simandiras, R. D. Amos, and N. C. Handy, Chem. Phys. 114, 9 (1987).

${ }^{46}$ P. Jørgensen and T. Helgaker, J. Chem. Phys. 89, 1560 (1988).

${ }^{47}$ T. Helgaker, P. Jørgensen, and N. C. Handy, Theor. Chim. Acta 76, 227 (1989).

${ }^{48}$ M. J. Frisch, M. Head-Gordon, and J. A. Pople, Chem. Phys. Lett. 166, 275 (1990).

${ }^{49}$ M. J. Frisch, M. Head-Gordon, and J. A. Pople, Chem. Phys. Lett. 166, 281 (1990).

${ }^{50}$ F. Haase and R. Ahlrichs, J. Comput. Chem. 14, 907 (1983).

${ }^{51}$ I. M. B. Nielsen, Chem. Phys. Lett. 255, 210 (1996).

${ }^{52}$ G. D. Fletcher, A. P. Rendell, and P. Sherwood, Mol. Phys. 91, 431 (1997).

${ }^{53}$ M. Head-Gordon, Mol. Phys. 96, 673 (1999).
${ }^{54}$ C. M. Aikens, S. P. Webb, R. L. Bell, G. D. Fletcher, M. W. Schmidt, and M. S. Gordon, Theor. Chim. Acta 110, 233 (2003).

${ }^{55}$ K. Ishimura, P. Pulay, and S. Nagase, J. Comput. Chem. 28, 2034 (2007).

${ }^{56}$ M. Feyereisen, G. Fitzgerald, and A. Komornicki, Chem. Phys. Lett. 208, 359 (1993).

${ }^{57}$ O. Vahtras, J. Almlöf, and M. Feyereisen, Chem. Phys. Lett. 213, 514 (1993).

${ }^{58}$ F. Weigend and M. Häser, Theor. Chim. Acta 97, 331 (1997).

${ }^{59}$ Y. M. Rhee, R. A. DiStasio, R. C. Lochan, and M. Head-Gordon, Chem. Phys. Lett. 426, 197 (2006).

${ }^{60}$ R. A. DiStasio, R. P. Steele, Y. M. Rhee, Y. Shao, and M. Head-Gordon, J. Comput. Chem. 28, 839 (2007).

${ }^{61}$ S. Kossmann and F. Neese, J. Chem. Theory Comput. 6, 2325 (2010).

${ }^{62}$ S. Schweizer, B. Doser, and C. Ochsenfeld, J. Chem. Phys. 128, 154101 (2008).

${ }^{63}$ J. Almlöf, Chem. Phys. Lett. 181, 319 (1991).

${ }^{64}$ A. E. Azhary, G. Rauhut, P. Pulay, and H.-J. Werner, J. Chem. Phys. 108, 5185 (1998).

${ }^{65}$ M. Schütz, H.-J. Werner, R. Lindh, and F. R. Manby, J. Chem. Phys. 121, 737 (2004).

${ }^{66}$ T. Nagata, D. G. Fedorov, K. Ishimura, and K. Kitaura, J. Chem. Phys. 135, $044110(2011)$

${ }^{67}$ C. A. White, B. G. Johnson, P. M. W. Gill, and M. Head-Gordon, Chem. Phys. Lett. 230, 8-16 (1994).

${ }^{68}$ C. Ochsenfeld and M. Head-Gordon, Chem. Phys. Lett. 270, 399 (1997).

${ }^{69}$ C. Ochsenfeld, C. A. White, and M. Head-Gordon, J. Chem. Phys. 109, 1663-1669 (1998).

${ }^{70}$ S. Coriani, S. Høst, B. Jansík, L. Thøgersen, J. Olsen, P. Jørgensen, S. Reine, F. Pawłowski, T. Helgaker, and P. Sałek, J. Chem. Phys. 126, 154108 (2007).

${ }^{71}$ C. Ochsenfeld, Chem. Phys. Lett. 327, 216-223 (2000).

${ }^{72}$ Y. Shao, C. A. White, and M. Head-Gordon, J. Chem. Phys. 114, 6572-6577 (2001).

${ }^{73}$ S. Reine, A. Krapp, M. F. Iozzi, V. Bakken, T. Helgaker, F. Pawłowski, and P. Sałek, J. Chem. Phys. 133, 044102 (2010).

${ }^{74}$ T. Helgaker, P. Jørgensen, and J. Olsen, Molecular Electronic Structure Theory, 1st ed. (Wiley, 2000).

${ }^{75}$ T. Helgaker and J. Almlöf, Int. J. Quantum Chem. 26, 275 (1984).

${ }^{76}$ J. Olsen, K. L. Bak, K. Ruud, T. Helgaker, and P. Jørgensen, Theor. Chim. Acta 90, 421 (1995).

${ }^{77}$ P. Jørgensen and J. Simons, J. Chem. Phys. 79, 334 (1983).

${ }^{78}$ D. Usvyat and M. Schütz, Theor. Chem. Acc. 114, 276 (2005).

${ }^{79}$ T. H. Dunning, Jr., J. Chem. Phys. 90, 1007-1023 (1989).

${ }^{80}$ LSDALTON, a linear scaling molecular electronic structure program, Release Dalton2011 (2011), see http://daltonprogram.org. 